\title{
Vison-generated photon mass in quantum spin ice: A theoretical framework
}

\author{
M. P. Kwasigroch (1) \\ London Centre for Nanotechnology, University College London, 17-19 Gordon Street, London WC1H 0AH, United Kingdom and \\ T.C.M. Group, Cavendish Laboratory, University of Cambridge, J. J. Thomson Avenue, Cambridge CB3 OHE, United Kingdom
}

(Received 31 October 2019; accepted 17 June 2020; published 10 September 2020)

\begin{abstract}
Describing the experimental signatures of quantum spin ice has been the focus of many theoretical efforts, as definitive experimental verification of this candidate quantum spin liquid is yet to be achieved. Gapped excitations known as visons have largely eluded those efforts. We provide a theoretical framework, which captures their dynamics and predicts new signatures in the magnetic response. We achieve this by studying the ring-exchange Hamiltonian of quantum spin ice in the large- $s$ approximation, taking into account the compact nature of the emergent U(1) gauge theory. We find the stationary solutions of the action - the instantons-which correspond to visons tunneling between lattice sites. By integrating out the instantons, we calculate the effective vison Hamiltonian, including their mass. We show that in the ground state virtual vison pairs simply renormalize the speed of light and give rise to an inelastic continuum of excitations. At low temperatures, however, thermally activated visons form a Debye plasma and introduce a mass gap in the photon spectrum, equal to the plasma frequency, which we calculate as a function of temperature. We demonstrate that this dynamical mass gap should be visible in energy-resolved neutron scattering spectra but not in the energy-integrated ones. We also show that it leads to the vanishing of the susceptibility of an isolated system, through a mechanism analogous to the Meissner effect, but that it does not lead to confinement of static spinons.
\end{abstract}

DOI: $10.1103 /$ PhysRevB.102.125113

\section{INTRODUCTION}

Quantum spin ice (QSI) is a candidate quantum spin liquid [1], where an emergent U(1) gauge symmetry prevents magnetic order down to zero temperature and gives rise to gauge and fractionalized excitations: a gapless photon, magnetic monopoles (spinons) and emergent electric charges (visons). In spite of this long list of predicted exotic excitations, definitive experimental verification is still missing, and this situation is shared by QSI with many other proposed spin liquids [2]. Hence, it is important for theoretical efforts to characterize as many signatures of these excitations as possible. In particular, the theoretical description of the inelastic response, through the dynamical structure factor, has recently been successful in providing evidence of a potential spin liquid in $\alpha-\mathrm{RuCl}_{3}$ [3-8].

In our previous work [9], we analytically characterized the photon excitation of QSI by including quantum fluctuations around the classical limit via a large-s expansion. We were also able to calculate the energy of the gapped vison excitation, but were not able to study its dynamics. In this paper, we extend the semiclassical description of our previous work to capture the vison dynamics and its contribution to the inelastic magnetic response of QSI.

The vison is an emergent excitation of QSI, a consequence of the compact U(1) gauge symmetry [10]. (The gauge group is compact because of the quantization of the spins.) Visons are sources of flux of the emergent electric field. Even though the field is divergenceless, a Dirac string carrying flux that is a multiple of $2 \pi$ has zero energy in a compact theory, allowing the charges at its ends to behave as free excitations. The state of these electric charges has profound consequences on the force between the magnetic charges (spinons). In particular, potential condensation of electric charges can lead to the confinement of magnetic charges.

It is believed that at zero temperatures visons simply lead to the renormalization of the speed of light, but at nonzero temperatures, it is anticipated that their effects are less benign. Reference [1] already drew comparisons with $(2+1)$ dimensional compact lattice gauge theory, which is always in the confined phase and has a gapped photon spectrum [11]. At increasing temperatures, the $(3+1)$-dimensional QSI becomes more like a $(2+1)$-dimensional compact lattice gauge theory and perhaps a condensation of visons leads to a photon mass gap and confinement of magnetic monopoles.

Despite their importance, so far, few experimental signatures of visons have been predicted [12,13]. They are sources of a fictitious electric field and hence cannot easily be probed directly in experiments. They have also largely eluded theoretical efforts of quantum Monte Carlo [14,16-20], due to the limited resolution of obtained excitation spectra, as well as mean-field treatments [21-25]. In this context, the work of Ref. [26], made recent progress by looking at visons through classical Monte Carlo, characterising their effect on emergent field correlators and the heat capacity. This work analysed vison signatures in the classical $s=\infty$ limit. Here, we will show that including nonperturbative corrections in $s$, makes the vison inertia finite and allows us to study their quantum dynamics at low temperatures. We demonstrate that these dynamics couple to the magnetic response of QSI via electromagnetic induction, which can be probed in neutron scattering experiments.

In our theoretical analysis, we are motivated by the success of our previous work [9], where we used a large-s semiclassical description to successfully capture the emergent 
electrodynamics of the ring-exchange Hamiltonian [10] and obtained a photon spectrum that was in good quantitative agreement with quantum Monte Carlo calculations for $s=$ $1 / 2$ [14]. As in our previous work, we map the ring-exchange Hamiltonian in the large-s limit onto compact U(1) lattice gauge theory using the Villain spin representation. However, this time we do not neglect spin quantization, or equivalently, the periodic nature of the emergent dual field, which allows us to include the dynamics of visons in the theoretical description. We show that, in the ground state the effects of these charges are rather innocuous; they exist as virtual, tightly bound pairs and simply renormalize the speed of light. At nonzero temperatures, however, they enter into existence as physical, thermally activated excitations, that form a plasma. We show that propagation of the photon through the vison plasma will appear gapped, which can be probed in neutron scattering experiments. We calculate typical scattering intensities that might be observed. We note that a similar effect had been anticipated in the context of hot QED by Ref. [27].

The paper is structured as follows. In Sec. II, we present the ring-exchange Hamiltonian in the Villain spin representation, which was introduced in our previous work. We then move to the dual electric charge representation, where the visons are made more manifest. We conclude the section by writing down the imaginary-time action of our model and finding its normal modes. In Sec. III, we analyze the stationary solutions of the action- the instantons-which correspond to the quantum tunneling of visons between neighboring lattice sites. In Sec. IV, we look at the ground state properties of our model. Sec. V studies the effects of visons at nonzero temperatures and presents the main results of this paper. Finally, in Sec. VI, we summarize all our main findings.

\section{THE MODEL}

Following on from our previous work [9], we study the ring-exchange Hamiltonian of QSI [10] in the Villain spin representation [28]

$$
\hat{H}=g \sum_{\alpha \beta}\left[e^{i \operatorname{cur}_{\alpha \beta} \hat{\phi} / 2} \prod_{i j \in \alpha \beta}\left(\tilde{s}^{2}-\hat{S}_{i j}^{z}\right)^{\frac{1}{2}} e^{i \operatorname{cur}_{\alpha \beta} \hat{\phi} / 2}+\text { H.c. }\right],
$$

where the spin azimuthal angle $\phi \in(-\pi, \pi]$, its projection on the $z$-axis $S^{z}$ is an integer or half-integer with $\left|S^{z}\right| \leqslant s$, $\left[\hat{\phi}, \hat{S^{z}}\right]=i, \tilde{s}=s+\frac{1}{2}$, and the product is over all six pyrochlore lattice sites $i j$ belonging to the plaquette centered on site $\alpha \beta$ of the dual pyrochlore lattice. The curl is taken around this plaquette. Just in like our previous work, Latin letters $\{i\}$ index the sites of the diamond lattice (bond midpoints $\{i j\}$ correspond to pyrochlore lattice sites on which the spins live) and the Greek letters index the sites of the dual diamond lattice (bond midpoints $\{\alpha \beta\}$ correspond to the dual pyrochlore lattice sites on which the plaquettes are centered). The spins satisfy the constraint

$$
\operatorname{div}_{i} S^{z} \equiv \sum_{i j}^{(i)} S_{i j}^{z}=Q_{i}
$$

where the sum is taken over the four pyrochlore lattice sites $i j$ that belong to the diamond lattice site $i$, i.e., over the four corners of the tetrahedron centered on $i$ (note that a positive sign is taken for "up" tetrahedra and negative for "down" tetrahedra). $Q_{i} \in \mathbb{Z}$ are static magnetic charges (magnetic monopoles) introduced into the system. The charges are static because the constraint commutes with the Hamiltonian and $Q_{i}$ are therefore constants of motion.

\section{A. Electric charge representation}

The visons are made more manifest in the dual, electric charge representation of the Hamiltonian. Rather than working with the conjugate magnetic field $\hat{S}_{i j}^{z}$ and the electric vector potential $\hat{\phi}_{i j}$, we shall be working with the conjugate electric field $E_{\alpha \beta}$ and the magnetic vector potential $A_{\alpha \beta}$. We introduce the new conjugate operators via

$$
\begin{aligned}
\hat{E}_{\alpha \beta} & =\operatorname{curl}_{\alpha \beta} \hat{\phi}, \\
\hat{S}_{i j}^{z} & =\operatorname{curl}_{i j} \hat{A}+\Delta_{i j} \psi,
\end{aligned}
$$

where $\psi_{i}$ is a scalar field (not an operator) defined on diamond lattice sites with $\Delta_{i j} \psi \equiv \psi_{j}-\psi_{i}$. Note that, because the electric field $E_{\alpha \beta}$ is defined as the lattice curl it necessarily has zero divergence. Equation (4) is just the lattice Helmholtz decomposition for the magnetic field and because its divergenceful part is a constant of motion, it can be expressed as $\Delta_{i j} \psi$ with $\psi_{i}$ a scalar field. The commutation relations for the magnetic $\hat{S}_{i j}^{z}$ and electric field $\hat{E}_{\alpha \beta}$ operators (which follow from $\left[\hat{\phi}_{i j}, S_{i j}^{z}\right]=i$ ) imply that the new conjugate operators have the canonical commutator $\left[\hat{E}_{\alpha \beta}, \hat{A}_{\alpha \beta}\right]=-i$.

Because the Hamiltonian is periodic in $\hat{E}_{\alpha \beta}$, the noninteger part of $\hat{A}_{\alpha \beta}, A_{\alpha \beta}^{0}$ is a constant of motion and is analogous to crystal momentum. It is therefore useful to make the following replacement:

$$
\hat{A}_{\alpha \beta} \rightarrow \hat{A}_{\alpha \beta}+A_{\alpha \beta}^{0},
$$

where the new operators $\hat{A}_{\alpha \beta}$ have strictly integer eigenvalues. The energy eigenstates, which are analogous to Bloch states, are now given by

$$
\begin{aligned}
|\Psi\rangle & =\sum_{A_{\alpha \beta}} c_{A_{\alpha \beta}}\left|A_{\alpha \beta}\right\rangle \otimes\left|A_{\alpha \beta}^{0}\right\rangle \\
& =\int d E_{\alpha \beta} \Psi\left(E_{\alpha \beta}\right)\left|E_{\alpha \beta}\right\rangle \otimes\left|A_{\alpha \beta}^{0}\right\rangle,
\end{aligned}
$$

where $\Psi\left(E_{\alpha \beta}\right)$ is a periodic function of $E_{\alpha \beta}$ and the kets are eigenstates of the respective operators. After the above replacement, the magnetic field becomes

$$
\hat{S}_{i j}^{z}=\operatorname{curl}_{i j} \hat{A}_{\alpha \beta}+B_{i j}^{0},
$$

where $B_{i j}^{0}$ is a static background field

$$
B_{i j}^{0}=\operatorname{curl}_{i j} A_{\alpha \beta}^{0}+\Delta_{i j} \psi .
$$

Different constraints on the allowed values of the magnetic field $\hat{S}_{i j}^{z}$ can be implemented as constraints on the allowed eigenstates of $\hat{A}_{\alpha \beta}$ and values of the static background field $B_{i j}^{0}$. For instance, to realize the constraint that the magnetic field is half-integer valued we simply choose any background 
field $A_{\alpha \beta}^{0}$ that is half-integer valued. The field $\hat{A}_{\alpha \beta}$ then becomes unconstrained. The kinematic constraint $\left|S_{i j}^{z}\right| \leqslant s$ is the hardest to implement, since for any background field $A_{\alpha \beta}^{0}$, there are forbidden eigenstates of $\hat{A}_{\alpha \beta}$. As discussed in our previous work [9], in the large-s limit, the typical fluctuations $S_{i j}^{z} \sim \sqrt{s}$ and hence this constraint is largely irrelevant.

Any magnetic charges $Q_{i}$ introduced into the system [see Eq. (2)] uniquely determine $\psi_{i}$ via the lattice Laplace equation

$$
\operatorname{div}_{i} B^{0} \equiv \nabla_{i}^{2} \psi=Q_{i},
$$

where $\nabla_{i}^{2}$ is the lattice Laplacian. To realize the constraint that the magnetic field is integer (half-integer) valued, we must also choose a configuration $A_{\alpha \beta}^{0}$ that makes $B_{i j}^{0}$ integer (halfinteger) - this is important for confinement and is analysed in detail in Appendix F.

Because energy eigenstates are periodic in $E_{\alpha \beta}$, without loss of generality, we will restrict $E_{\alpha \beta} \in(-\pi, \pi]$, i.e., measure the field modulo $2 \pi$. The electric field now acquires a nonzero divergence quantized in units of $2 \pi$

$$
\operatorname{div}_{\alpha} \hat{E} \equiv \sum_{\alpha \beta}^{(\alpha)} \hat{E}_{\alpha \beta}=2 \pi q_{\alpha},
$$

where $q_{\alpha} \in \mathbb{Z}$ are the emergent electric charges $((\alpha)$ identifies that the sum is taken over the four corners of the tetrahedron $\alpha$ of the dual diamond lattice and again positive sign is taken for "up" tetrahedra and negative for "down" tetrahedra). A single electric charge at the tetrahedron $\alpha\left(q_{\alpha}= \pm 1\right)$ gives rise to a smooth, long-wavelength modulation of the electric field $E_{\alpha \beta} \propto \frac{1}{R^{2}}$, where $R$ is the distance from the electric charge. This long-wavelength modulation is a gapped topological excitation known as the vison.

To summarize this subsection, we write down the ringexchange Hamiltonian in the electric charge representation

$$
\begin{aligned}
\hat{H}=-g \sum_{\alpha \beta} & \left\{e^{i \hat{E}_{\alpha \beta} / 2} \prod_{i j \in \alpha \beta}\left[\tilde{s}^{2}-\left(\operatorname{curl}_{i j} \hat{A}+B_{i j}^{0}\right)^{2}\right]^{\frac{1}{2}}\right. \\
& \left.\times e^{i E_{\alpha \beta} / 2}+\text { H.c. }\right\} .
\end{aligned}
$$

We will be working in the $s \gg 1$ limit and expand the Hamiltonian accordingly

$$
\hat{H}=\tilde{g} \sum_{\alpha \beta} \hat{E}_{\alpha \beta}^{2}+\sum_{i j} \frac{\tilde{g} z}{s^{2}}\left(\operatorname{curl}_{i j} \hat{A}+B_{i j}^{0}\right)^{2}+\mathcal{O}\left(s^{-2}\right),
$$

where $\tilde{g}=g s^{6}$ and $z=6$. We have also replaced $\tilde{s}$ with $s$, as we will be working to the first nonvanishing order in $s$ for all physical quantities that we calculate.

\section{B. Partition function}

To study the nonperturbative effects of dynamical electric charges, we begin with the partition function for the above Hamiltonian, obtained in the usual way by inserting two resolutions of the identity in the $\hat{E}_{\alpha \beta}$ and $\hat{A}_{\alpha \beta}$ bases into each of the $N_{\tau}$ Suzuki-Trotter time slices (the time slice width is given by $\epsilon=\frac{\beta}{N_{\tau}}$ ).

$$
\begin{gathered}
\mathcal{Z}=\prod_{\tau, \alpha \beta, \gamma} \int_{-\pi}^{\pi} d E_{\alpha \beta}(\tau) \int_{-\infty}^{+\infty} d A_{\alpha \beta}(\tau) \int_{-\infty}^{+\infty} d \varphi_{\gamma}(\tau) \\
\times \sum_{j \alpha \beta} e^{-\mathcal{S}} \\
\mathcal{S}=\sum_{\tau}\left\{i \sum_{\alpha \beta} A_{\alpha \beta}(\tau) \Delta_{\tau} E_{\alpha \beta}(\tau)+\epsilon \tilde{g} \sum_{\alpha \beta} E_{\alpha \beta}^{2}(\tau)\right. \\
+\epsilon \frac{\tilde{g} z}{s^{2}} \sum_{i j}\left[\operatorname{curl}_{i j} A(\tau)+B_{i j}^{0}\right]^{2} \\
\left.+\epsilon \sum_{\gamma} i \varphi_{\gamma}(\tau)\left[\operatorname{div}_{\gamma} E(\tau)-2 \pi q_{\gamma}(\tau)\right]\right\} \\
+\sum_{\tau, \alpha \beta} 2 \pi i j_{\alpha \beta}(\tau) A_{\alpha \beta}(\tau),
\end{gathered}
$$

where $\Delta_{\tau} E_{\alpha \beta} \equiv E_{\alpha \beta}(\tau+\epsilon)-E_{\alpha \beta}(\tau)$, the sum over integers $j_{\alpha \beta}(\tau)$ ensures that $A_{\alpha \beta}(\tau)$ are integer-valued, and $\varphi_{\gamma}(\tau)$ are Lagrange multipliers that ensure the divergence of the electric field at each dual diamond lattice site $\gamma$ is an integer multiple $q_{\gamma}$ of $2 \pi$ for all $\tau$. The Lagrange multipliers can be interpreted as the scalar electric potential.

Note that the zero modes, arising from the $\mathrm{U}(1)$ gauge symmetry of the action, $A_{\alpha \beta}(\tau) \rightarrow A_{\alpha \beta}(\tau)+\chi_{\beta}(\tau)-\chi_{\alpha}(\tau)$, $\varphi_{\gamma}(\tau) \rightarrow \varphi_{\gamma}(\tau)+\chi_{\gamma}(\tau+\epsilon)-\chi_{\gamma}(\tau)$ enforce vison charge conservation

$$
\Delta_{\tau} q_{\gamma}+\operatorname{div}_{\gamma} j=0
$$

for all tetrahedra $\gamma$. We can see that $j_{\alpha \beta}(\tau)$ can be interpreted as the vison current between the tetrahedra touching at $\alpha \beta$.

\section{Normal modes of the action}

We first conveniently parametrize the sites of the dual pyrochlore lattice. The dual pyrochlore lattice is a superposition of four fcc lattices indexed by $\mu=1,2,3$, and 4 . Taking a single up tetrahedron from the dual diamond lattice, with its center located at the position vector $\mathbf{r}$, the position vectors of its four corners $(\mu=1,2,3,4)$ are $\left(\mathbf{r}+\mathbf{e}_{\mu} / 2\right)$, where the four basis vectors $\mathbf{e}_{\mu}$ are given by

$$
\begin{aligned}
& \mathbf{e}_{1}=\frac{a_{0}}{4}(1,1,1), \quad \mathbf{e}_{2}=\frac{a_{0}}{4}(1,-1,-1), \\
& \mathbf{e}_{3}=\frac{a_{0}}{4}(-1,1,-1), \quad \mathbf{e}_{4}=\frac{a_{0}}{4}(-1,-1,1) .
\end{aligned}
$$

The centres of all up tetrahedra map out an fcc lattice. Each $\mu$ fcc lattice is then a translation of this lattice by $\mathbf{e}_{\mu} / 2$ and corresponds to the set of all those up tetrahedra corners that are displaced by $\mathbf{e}_{\mu} / 2$ from their centres. We thus identify each dual pyrochlore lattice site $\alpha \beta$ by an index $\mu$, corresponding to the fcc lattice to which this site belongs, and its position vector on that fcc lattice. This is reflected in the following change of notation for the variables

$$
\begin{aligned}
E_{\alpha \beta}(\tau) & \rightarrow E_{\mu}\left(\mathbf{r}_{\alpha}+\mathbf{e}_{\mu} / 2, \tau\right), \\
A_{\alpha \beta}(\tau) & \rightarrow A_{\mu}\left(\mathbf{r}_{\alpha}+\mathbf{e}_{\mu} / 2, \tau\right), \\
j_{\alpha \beta}(\tau) & \rightarrow j_{\mu}\left(\mathbf{r}_{\alpha}+\mathbf{e}_{\mu} / 2, \tau\right),
\end{aligned}
$$


where $\mathbf{r}_{\alpha}+\mathbf{e}_{\mu} / 2$ is the position vector of the site $\alpha \beta$ and $\mu$ identifies the fcc lattice to which it belongs. $\mathbf{r}_{\alpha}$ and $\mathbf{r}_{\beta}$ are the position vectors of the up and down tetrahedra, respectively, which touch at the site $\alpha \beta$, and $\mathbf{e}_{\mu}=\mathbf{r}_{\beta}-\mathbf{r}_{\alpha}$.

We also take the continuum limit $\epsilon \rightarrow 0$ of $\mathcal{S}$, where

$$
\begin{aligned}
& \sum_{\tau} \epsilon \rightarrow \int_{-\beta / 2}^{\beta / 2} d \tau, \\
& \frac{\Delta_{\tau} E_{\alpha \beta}}{\epsilon} \rightarrow \dot{E}_{\alpha \beta}, \\
& \sum_{\tau} j_{\alpha \beta}(\tau) A_{\alpha \beta}(\tau) \\
& \quad \rightarrow \int_{-\beta / 2}^{\beta / 2} d \tau A_{\alpha \beta}(\tau)\left[\sum_{\tau_{0}} j_{\alpha \beta}\left(\tau_{0}\right) \delta\left(\tau-\tau_{0}\right)\right],
\end{aligned}
$$

and we will set the background field $B_{i j}^{0}$ to zero for now and return to analyzing its effects later.

Because $A_{\alpha \beta}(\tau)$ is a continuous variable, provided we enforce charge conservation in Eq. (14), we can fix its gauge via the usual Faddeev-Popov procedure [29]. We choose to work in the Coulomb gauge

$$
\operatorname{div}_{\alpha} A \equiv \sum_{\alpha \beta}^{(\alpha)} A_{\alpha \beta}=0,
$$

where the longitudinal and transverse parts of the electric field $E_{\alpha \beta}(\tau)$ decouple. (The longitudinal part of $E_{\alpha \beta}$ satisfies $\operatorname{curl}_{i j} E(\tau)=0$ everywhere, whereas the transverse part satisfies $\operatorname{div}_{\alpha} E(\tau)=0$ everywhere.) In the Coulomb gauge, the action can be decomposed as follows:

$$
\mathcal{S}=\mathcal{S}_{\text {long. }}+\mathcal{S}_{\text {tran. }},
$$

where $\mathcal{S}_{\text {long. }}$ is a functional of the longitudinal part of $E_{\alpha \beta}(\tau)$ only and $\mathcal{S}_{\text {tran. }}$ of the transverse part only.

\section{Transverse modes}

The transverse part of the action $\mathcal{S}_{\text {tran. }}$ is diagonal in the eigenbasis of the curl operator, which is defined as

$$
\operatorname{curl}_{i j} A \equiv \sum_{\nu \neq \mu, \pm} \pm A_{\nu}\left(\mathbf{r}_{i}+\mathbf{e}_{\mu} / 2 \pm \boldsymbol{\Delta}_{\mu \nu}, \tau\right)
$$

where

$$
\boldsymbol{\Delta}_{\mu \nu} \equiv \frac{a_{0}}{\sqrt{8}} \frac{\mathbf{e}_{\mu} \times \mathbf{e}_{v}}{\left|\mathbf{e}_{\mu} \times \mathbf{e}_{v}\right|},
$$

and $\mathbf{r}_{i}+\mathbf{e}_{\mu} / 2$ is the position vector of the plaquette center $i j$ on the original pyrochlore lattice. The decomposition for the original pyrochlore lattice works in the same way as that for the dual pyrochlore lattice: $\mathbf{r}_{i}$ gives the position vector of the center of the $i$ up tetrahedron, of the original diamond lattice, and centres of all up tetrahedra map out an fcc lattice, which can be translated by $\mathbf{e}_{\mu} / 2$ to give one of the four $\mu=1,2,3$, and $4 \mathrm{fcc}$ lattices the plaquette center $i j$ belongs to. We transform to the eigenbasis of the curl operator in the same way that we have done in our previous work [9] for the original pyrochlore lattice

$$
\begin{aligned}
& A_{\mu}\left(\mathbf{r}+\mathbf{e}_{\mu} / 2, \tau\right) \\
& \quad=\frac{1}{\sqrt{N_{s} \beta}} \sum_{\mathbf{k} \in \mathrm{BZ}, \omega, \lambda} U_{\mu \lambda}^{\dagger}(\mathbf{k}) A_{\lambda}(\mathbf{k}, \omega) e^{i \mathbf{k} \cdot\left(\mathbf{r}+\mathbf{e}_{\mu} / 2\right)-i \omega \tau}, \\
& E_{\mu}\left(\mathbf{r}+\mathbf{e}_{\mu} / 2, \tau\right) \\
& \quad=\frac{1}{\sqrt{N_{s} \beta}} \sum_{\mathbf{k} \in \mathrm{BZ}, \omega, \lambda} U_{\mu \lambda}^{\dagger}(\mathbf{k}) E_{\lambda}(\mathbf{k}, \omega) e^{i \mathbf{k} \cdot\left(\mathbf{r}+\mathbf{e}_{\mu} / 2\right)-i \omega \tau},
\end{aligned}
$$

where $\lambda=1,2,3$, and 4 index the normal modes of the action, the wave vectors $\mathbf{k}$ are summed over the first Brillouin zone of the fcc lattice, $\omega$ are Matsubara frequencies, $N_{s}$ is the number of sites of the fcc lattice, and $U_{\mu \lambda}^{\dagger}(\mathbf{k})$ are $4 \times 4$ unitary matrices. In this basis, the curl operator can be written as

$$
\begin{aligned}
\operatorname{curl}_{i j}(A)= & \frac{1}{\sqrt{N_{s} \beta}} \sum_{\mathbf{k} \in \mathrm{BZ}, \omega} \sum_{v, \lambda} Z_{\mu v}(\mathbf{k}) U_{\nu \lambda}^{\dagger}(\mathbf{k}) \\
& \times e^{i \mathbf{k} \cdot\left(\mathbf{r}_{\alpha}+\mathbf{e}_{\mu} / 2\right)-i \omega \tau} A_{\lambda}(\mathbf{k}, \omega) \\
= & \frac{1}{\sqrt{N_{s} \beta}} \sum_{\lambda, \mathbf{k} \in \mathrm{BZ}, \omega} \xi_{\lambda}(\mathbf{k}) U_{\mu \lambda}^{\dagger}(\mathbf{k}) \\
& \times e^{i \mathbf{k} \cdot\left(\mathbf{r}_{i}+\mathbf{e}_{\mu} / 2\right)-i \omega \tau} A_{\lambda}(\mathbf{k}, \omega),
\end{aligned}
$$

where the columns of the matrix $U_{\nu \lambda}^{\dagger}(\mathbf{k})$ are eigenvectors of the matrix $Z_{\mu \nu}(\mathbf{k})=2 i \sin \left(\mathbf{k} \cdot \boldsymbol{\Delta}_{\mu \nu}\right)$ with eigenvalues

$$
\begin{aligned}
& \xi_{\lambda=1,2}(\mathbf{k})= \pm \sqrt{2} \sqrt{\sum_{\mu \nu} \sin ^{2}\left(\mathbf{k} \cdot \boldsymbol{\Delta}_{\mu \nu}\right)}, \\
& \xi_{\lambda=3,4}(\mathbf{k})=0 .
\end{aligned}
$$

This identifies $\lambda=3$ and 4 as the longitudinal modes since they vanish under the action of the curl operator. It follows that the transverse part of the action becomes diagonal in the new basis

$$
\begin{aligned}
\mathcal{S}_{\text {tran. }}= & \sum_{\omega} \sum_{\mathbf{k} \in \mathrm{BZ}, \lambda=1,2}\left[\omega A_{\lambda}(-\mathbf{k},-\omega) E_{\lambda}(\mathbf{k}, \omega)\right. \\
& +\tilde{g} E_{\lambda}(-\mathbf{k},-\omega) E_{\lambda}(\mathbf{k}, \omega)+\frac{z \tilde{g}}{s^{2}} \xi_{\lambda}^{2}(\mathbf{k}) A_{\lambda}(-\mathbf{k},-\omega) \\
& \left.\times A_{\lambda}(\mathbf{k}, \omega)+2 \pi i j_{\lambda}(\mathbf{k}, \omega) A_{\lambda}(-\mathbf{k},-\omega)\right],
\end{aligned}
$$

where in the Coulomb gauge $A_{\lambda=3,4}(\mathbf{k}, \omega)=0$ and

$$
\begin{aligned}
j_{\lambda}(\mathbf{k}, \omega)= & \frac{1}{\sqrt{N_{s} \beta}} \sum_{\mu, \tau, \mathbf{r} \in \mathrm{fcc}} U_{\lambda \mu}(\mathbf{k}) j_{\mu}\left(\mathbf{r}+\mathbf{e}_{\mu} / 2, \tau\right) \\
& \times e^{i \omega \tau-i \mathbf{k} \cdot\left(\mathbf{r}+\mathbf{e}_{\mu} / 2\right)} .
\end{aligned}
$$

\section{Longitudinal modes}

We proceed to finding the normal modes of the longitudinal part of the action. The constraint in Eq. (10) uniquely determines the longitudinal ( $\lambda=3$ and 4$)$ modes of the electric field $E_{\alpha \beta}(\tau)$ via the lattice Laplace equation. To form the equation, we write the longitudinal field as the lattice derivative

$$
E\left(\mathbf{r}+\mathbf{e}_{\mu} / 2, \tau\right)=\Phi_{\mathrm{d}}\left(\mathbf{r}+\mathbf{e}_{\mu}, \tau\right)-\Phi_{\mathrm{u}}(\mathbf{r}, \tau),
$$


where the variables $\Phi_{\mathrm{u} / \mathrm{d}}(\mathbf{r})$ are defined on the centres of up/down tetrahedra that make up two fcc lattices. Making the above substitution, the constraint in Eq. (10), for the up tetrahedra, can be written as

$$
2 \pi q_{\mathrm{u}}(\mathbf{r}, \tau)=\sum_{\mu}\left[\Phi_{\mathrm{d}}\left(\mathbf{r}+\mathbf{e}_{\mu}, \tau\right)-\Phi_{\mathrm{u}}(\mathbf{r}, \tau)\right]
$$

and for the down tetrahedra as

$$
2 \pi q_{\mathrm{d}}(\mathbf{r}, \tau)=-\sum_{\mu}\left[\Phi_{\mathrm{d}}(\mathbf{r}, \tau)-\Phi_{\mathrm{u}}\left(\mathbf{r}-\mathbf{e}_{\mu}, \tau\right)\right],
$$

where $q_{\mathrm{u}}(\mathbf{r}, \tau)$ and $q_{\mathrm{d}}(\mathbf{r}, \tau)$ are the vison occupation numbers at position $\mathbf{r}$ of an up or down tetrahedron respectively. Fourier transforming the two equations we obtain a unique solution (up to a constant) for $\Phi_{\mathrm{u} / \mathrm{d}}(\mathbf{r}, \tau)$

$$
\left(\begin{array}{c}
\Phi_{\mathrm{u}}(\mathbf{k}, \tau) \\
\Phi_{\mathrm{d}}(\mathbf{k}, \tau)
\end{array}\right)=\frac{\pi}{2\left(|\gamma(\mathbf{k})|^{2}-1\right)}\left(\begin{array}{cc}
1 & \gamma(\mathbf{k}) \\
\gamma^{*}(\mathbf{k}) & 1
\end{array}\right)\left(\begin{array}{l}
q_{\mathrm{u}}(\mathbf{k}, \tau) \\
q_{\mathrm{d}}(\mathbf{k}, \tau)
\end{array}\right),
$$

where $\gamma(\mathbf{k})=\frac{1}{4} \sum_{\mu} e^{i \mathbf{k} \cdot \mathbf{e}_{\mu}}, \Phi_{\mathrm{u} / \mathrm{d}}(\mathbf{k})=\frac{1}{\sqrt{N_{s}}} \sum_{\mathbf{r} \in \mathrm{u} / \mathrm{d}} e^{-i \mathbf{k} \cdot \mathbf{r}} \Phi_{\mathrm{u} / \mathrm{d}}(\mathbf{r})$ and $q_{\mathrm{u} / \mathrm{d}}(\mathbf{k})=\frac{1}{\sqrt{N_{s}}} \sum_{\mathbf{r} \in \mathrm{u} / \mathrm{d}} e^{-i \mathbf{k} \cdot \mathbf{r}} q_{\mathrm{u} / \mathrm{d}}(\mathbf{r})(\mathbf{r}$ is summed over the positions of up/down tetrahedra, respectively). Substituting this back into $\mathcal{S}$ in Eq. (13), we obtain the unique longitudinal part of the action

$$
\begin{aligned}
\mathcal{S}_{\text {long. }}= & \int_{-\beta / 2}^{\beta / 2} d \tau\left[\sum_{\mathbf{r} \mathbf{r}^{\prime} \in \mathrm{u}} V\left(\mathbf{r}-\mathbf{r}^{\prime}\right) q_{\mathrm{u}}(\mathbf{r}, \tau) q_{\mathrm{u}}\left(\mathbf{r}^{\prime}, \tau\right)\right. \\
& +\sum_{\mathbf{r} \mathbf{r}^{\prime} \in \mathrm{d}} V\left(\mathbf{r}-\mathbf{r}^{\prime}\right) q_{\mathrm{d}}(\mathbf{r}, \tau) q_{\mathrm{d}}\left(\mathbf{r}^{\prime}, \tau\right) \\
& \left.+\sum_{\mathbf{r} \in \mathrm{u}} \sum_{\mathbf{r}^{\prime} \in \mathrm{d}} V_{\mathrm{ud}}\left(\mathbf{r}-\mathbf{r}^{\prime}\right) q_{\mathrm{u}}(\mathbf{r}, \tau) q_{\mathrm{d}}\left(\mathbf{r}^{\prime}, \tau\right)\right],
\end{aligned}
$$

where

$$
\begin{aligned}
V\left(\mathbf{r}-\mathbf{r}^{\prime}\right) & =\frac{\tilde{g}}{N_{s}} \sum_{\mathbf{k} \in \mathrm{BZ}} \frac{\pi^{2}}{1-|\gamma(\mathbf{k})|^{2}} e^{i \mathbf{k} \cdot\left(\mathbf{r}-\mathbf{r}^{\prime}\right)}, \\
V_{\mathrm{ud}}\left(\mathbf{r}-\mathbf{r}^{\prime}\right) & =\frac{\tilde{g}}{N_{s}} \sum_{\mathbf{k} \in \mathrm{BZ}} \frac{2 \pi^{2} \gamma(\mathbf{k})}{1-|\gamma(\mathbf{k})|^{2}} e^{i \mathbf{k} \cdot\left(\mathbf{r}-\mathbf{r}^{\prime}\right)} .
\end{aligned}
$$

Assuming the visons are far apart, it is useful to decompose the sum into its diagonal and off-diagonal parts

$$
\begin{aligned}
\mathcal{S}_{\text {long. }}= & \int_{-\beta / 2}^{\beta / 2} d \tau \mu_{\mathrm{V}} \sum_{\sigma=u / d, \mathbf{r} \in \mathrm{fcc}} q_{\sigma}^{2}(\mathbf{r}, \tau)+\int_{-\beta / 2}^{\beta / 2} d \tau \\
& \times \sum_{\sigma, \sigma^{\prime}, \mathbf{r}, \mathbf{r}^{\prime} \in \mathrm{fcc}} \tilde{V}\left(\mathbf{r}-\mathbf{r}^{\prime}\right) q_{\sigma}(\mathbf{r}, \tau) q_{\sigma^{\prime}}\left(\mathbf{r}^{\prime}, \tau\right),
\end{aligned}
$$

where $\tilde{V}(\mathbf{r})$ is the asymptotic Coulomb part of the vison interaction energy with the additional constraint that $\tilde{V}(\mathbf{0})=0$ and the vison chemical potential (self-energy) is given by

$$
\mu_{V}=\frac{\tilde{g}}{N_{s}} \sum_{\mathbf{k} \in \mathrm{BZ}} \frac{\pi^{2}}{1-|\gamma(\mathbf{k})|^{2}}=C_{1} \tilde{g}
$$

where $C_{1}=17.7$. For $\mathbf{r} \neq \mathbf{0}$,

$$
\tilde{V}(\mathbf{r})=\int \frac{d^{3} \mathbf{k}}{(2 \pi)^{3}} \frac{8 \tilde{g} a_{0} \pi^{2}}{|\mathbf{k}|^{2}} e^{i \mathbf{k} \cdot \mathbf{r}} .
$$

The above vison self-energy and Coulomb interaction agree with the results of Ref. [26] in the quadratic approximation. (Note that our definition of $g$ and the definition given in Ref. [26] differ by a factor of 2.)

\section{INSTANTONS}

\section{A. Stationary solutions of the action}

Stationary solutions of the Euclidean action make an important nonperturbative $\left(\propto e^{-s}\right)$ contribution to the partition function, that restores the periodic symmetry with respect to the electric field $E_{\alpha \beta}(\tau)$. The instanton is precisely such a stationary solution. For illustration, let us first consider a single instanton at $\mathbf{r}=\mathbf{0}$ and imaginary time $\tau=0$ in the direction $\mathbf{e}_{\sigma}$, i.e., $j_{\mu=\sigma}(\mathbf{0}, 0)=1$ with all other $j_{\alpha \beta}$ vanishing. To satisfy the continuity equation in Eq. (14), we will consider a vison of charge $2 \pi$ tunneling from the up tetrahedron at $\mathbf{r}=-\mathbf{e}_{\sigma} / 2$ to the down tetrahedron at $\mathbf{r}=\mathbf{e}_{\sigma} / 2$. Correspondingly, the vison occupation number $q_{\mathrm{u}}\left(-\mathbf{e}_{\sigma} / 2, \tau\right)\left[q_{\mathrm{d}}\left(\mathbf{e}_{\sigma} / 2, \tau\right)\right]$ decreases (increases) at $\tau=0$ by one. (Alternatively, we could have considered a vison of charge $-2 \pi$ tunneling in the opposite direction or a creation of a vison-antivison pair.)

When deriving the stationary solution of the action, it proves convenient to briefly reinstate the longitudinal part of $A_{\alpha \beta}(\tau)\left[A(\mathbf{k}, \omega)_{\lambda=3,4}\right.$ normal modes $]$ in the action so that the continuity expressed in Eq. (14) is automatically taken care of. (This, of course, has no effect on the physical, gauge-invariant observables and is the only place where we are not working in the Coulomb gauge.) We can see how continuity is enforced as follows. Substituting the longitudinal part of $A_{\alpha \beta}(\tau)$, which can be written as $A_{\alpha \beta}(\tau)=\chi_{\beta}(\tau)-\chi_{\alpha}(\tau)$, into the action $\mathcal{S}$, and collecting all terms where it enters, we obtain

$$
-i \sum_{\alpha, \tau} \chi_{\alpha}(\tau)\left[\operatorname{div}_{\alpha} \Delta_{\tau} E(\tau)+2 \pi \operatorname{div}_{\alpha} j(\tau)\right] .
$$

The longitudinal part $\chi_{\alpha}(\tau)$ can thus be interpreted as a Lagrange multiplier that enforces the continuity expressed in Eq. (14). The instanton solution can now be derived from the transverse part of the action in Eq. (25) by extending the sum to include the longitudinal modes $\lambda=3,4$. Minimising $\mathcal{S}$ in Eq. (25) with respect to variations in the variables $E_{\lambda}(\mathbf{k}, \omega)$ and $A_{\lambda}(\mathbf{k}, \omega)$, we obtain the following equations of motion

$$
\begin{aligned}
& \omega A_{\lambda}(\mathbf{k}, \omega)=2 \tilde{g} E_{\lambda}(\mathbf{k}, \omega), \\
& \omega E_{\lambda}(\mathbf{k}, \omega)+\frac{2 z \tilde{g}}{s^{2}} \xi_{\lambda}^{2}(\mathbf{k}) A_{\lambda}(\mathbf{k}, \omega)+\frac{2 \pi i}{\sqrt{N_{s} \beta}} U_{\lambda \sigma}(\mathbf{k})=0 .
\end{aligned}
$$

Solving for the electric field, we obtain the instanton solution

$$
E_{\lambda}^{\text {inst. }}(\mathbf{k}, \omega)=E_{\lambda}^{\text {const. }}(\mathbf{k}) \delta_{\omega, 0}-\frac{1}{\sqrt{N_{s} \beta}} \frac{\pi i \omega U_{\lambda \sigma}(\mathbf{k})}{\frac{2 z \tilde{g}^{2}}{s^{2}} \xi_{\lambda}^{2}(\mathbf{k})+\frac{\omega^{2}}{2}},
$$

where $E_{\lambda}^{\text {const. }}(\mathbf{k})$ is constant in time and is a purely divergenceful field [i.e., $E_{\lambda=1,2}^{\text {const. }}(\mathbf{k})=0$ ] due to a pair of visons of charge $\pi$ at positions $\pm \mathbf{e}_{\mu} / 2$. This is to ensure that the longitudinal part of $E_{\mu}^{\text {inst. }}(\mathbf{r}, \tau)$ is the electric field due to a vison of charge $2 \pi$, at $\mathbf{r}=-\mathbf{e}_{\sigma} / 2$ for $\tau<0$, and at $\mathbf{r}=\mathbf{e}_{\sigma} / 2$ for $\tau>0$. (See Appendix A for further details.) 
It is important to check that our solution lies within the domain $\left|E_{\alpha \beta}(\tau)\right| \leqslant \pi$. By symmetry, the solution will reach its maximum at $\tau=0, \mathbf{r}=\mathbf{0}$

$$
\begin{aligned}
E_{\sigma}^{\text {inst. }}(\mathbf{0}, 0) & =\int_{-\infty}^{\infty} \frac{d \omega}{2 \pi N_{s}} \sum_{\lambda, \mathbf{k} \in \mathrm{BZ}} \frac{-\pi i \omega U_{\sigma \lambda}^{\dagger}(\mathbf{k}) U_{\lambda \sigma}(\mathbf{k})}{\frac{2 z \tilde{g}^{2}}{s^{2}} \xi_{\lambda}^{2}(\mathbf{k})+\frac{\omega^{2}}{2}} \\
& = \pm \pi
\end{aligned}
$$

where the field jumps from $-\pi$ to $\pi$ at $\tau=0$ and we have used the fact that $E_{\mu}^{\text {const. }}(\mathbf{r}=\mathbf{0})=0$. We have thus verified that $\left|E_{\alpha \beta}(\tau)\right| \leqslant \pi$ everywhere. Note that we are working in the low-temperature limit $\beta / \tau_{\mathrm{QF}} \gg 1$, where finite-size effects in imaginary time can be neglected and $\sum_{\omega \in 2 \pi n / \beta} \approx$ $\int_{-\infty}^{\infty} \beta \frac{d \omega}{2 \pi} \cdot \tau_{\mathrm{QF}}=\frac{s}{\tilde{g} \sqrt{z}}$ is the characteristic timescale of quantum fluctuations. Notice that $\beta / \tau_{\mathrm{QF}} \gg 1$ implies that the temperatures are low by comparison with the photon bandwidth [9].

\section{B. The instanton measure}

To compute the measure associated with a single instanton, we first remove the discontinuity in the instanton solution $E_{\mu}^{\text {inst. }}(\mathbf{r}, \tau)$ at $\tau=0$ [see Eq. (38)] and let $E_{\sigma}(\mathbf{0}, \tau)$ wind by $2 \pi$ instead

$$
E_{\mu}(\mathbf{r}, \tau) \rightarrow E_{\mu}(\mathbf{r}, \tau)-\delta_{\mathbf{r}, 0} \delta_{\mu \sigma}[\pi \operatorname{sgn}(\tau)+\pi] .
$$

Note that this transformation removes the jump in the longitudinal part of $E_{\mu}^{\text {inst. }}(\mathbf{r}, \tau)$ that occurs at $\tau=0$ (see Appendix B). The longitudinal part is now the electric field due to a vison of charge $2 \pi$ at $\mathbf{r}=-\mathbf{e}_{\sigma} / 2$ for $\tau>0$ as well as $\tau<0$. The longitudinal part is thus a constant and the dynamical part is purely transverse: $(\lambda=1,2)$ are the only components we need to consider, when calculating the instanton measure. Considering the action $\mathcal{S}$ in Eq. (13), the above transformation removes the $2 \pi i j_{\sigma}(\mathbf{0}, 0) A_{\sigma}(\mathbf{0}, 0)$ term, translates the quadratic term $E_{\sigma}^{2}(\mathbf{0}, \tau>0)$ by $2 \pi$, alters the constraint enforced by $\varphi_{\alpha}(\tau)$ so that $\operatorname{div} E=2 \pi$ at $\mathbf{r}=\mathbf{e}_{\mu} / 2$ for all $\tau$, and changes the range of integration over $E_{\sigma}(\mathbf{0}, \tau)$ from $\left|E_{\sigma}(\mathbf{0}, \tau)\right| \leqslant \pi$ to $\pi \operatorname{sgn}(\tau)<E_{\sigma}(\mathbf{0}, \tau) \leqslant$ $2 \pi+\pi \operatorname{sgn}(\tau)$ (the range of integration over the other $E_{\alpha \beta}(\tau)$ variables remains unaltered). Integrating out the variables $A_{\lambda=1,2}(\mathbf{k}, \omega)$ in the transverse part of the action $\mathcal{S}_{\text {tran. }}$, we are left with the following action for the variables $E_{\lambda=1,2}(\mathbf{k}, \omega)$ together with a smooth instanton solution $E_{\mu}^{\mathrm{sm} .}(\mathbf{r}, \tau)$

$$
\begin{aligned}
& \mathcal{S}_{\operatorname{tran}}\left[E_{\lambda}(\mathbf{k}, \tau)\right]=\int_{-\beta / 2}^{\beta / 2} d \tau {\left[\sum_{\mathbf{k} \in \mathrm{BZ}, \lambda=1,2} \frac{s^{2}\left|\dot{E}_{\lambda}(\mathbf{k}, \tau)\right|^{2}}{4 z \tilde{g} \xi_{\lambda}^{2}(\mathbf{k})}\right.} \\
&\left.+\tilde{g} \sum_{\alpha \beta} V\left(E_{\alpha \beta}(\tau)\right)\right], \\
& E_{\mu}^{\text {sm. }}(\mathbf{r}, \tau)=E_{\mu}^{\text {inst. }}(\mathbf{r}, \tau)+\delta_{\mathbf{r}, 0} \delta_{\mu \sigma}[\pi \operatorname{sgn}(\tau)+\pi],
\end{aligned}
$$

where $V\left(E_{\alpha \beta}(\tau)\right)=\min _{n}\left(E_{\alpha \beta}(\tau)-2 \pi n\right)^{2}$ is a continued parabolic potential and the minimum is taken with respect to integer $n$. See Appendix B for verification that $E_{\mu}^{\mathrm{sm} .}(\mathbf{r}, \tau)$ is indeed a stationary solution of the above action.

Fluctuations around the smooth instanton solution $\delta E_{\mu}(\mathbf{r}, \tau)=E_{\mu}(\mathbf{r}, \tau)-E_{\mu}^{\mathrm{sm} .}(\mathbf{r}, \tau)$ are also smooth and are governed by the following action:

$$
\begin{aligned}
\delta \mathcal{S}_{\operatorname{tran}}\left[\delta E_{\lambda}(\mathbf{k}, \tau)\right]= & \int_{-\frac{\beta}{2}}^{\frac{\beta}{2}} d \tau \sum_{\mathbf{k} \in \mathrm{BZ}, \lambda=1,2} \frac{s^{2}\left|\delta \dot{E}_{\lambda}(\mathbf{k}, \tau)\right|^{2}}{4 z \tilde{g} \xi_{\lambda}^{2}(\mathbf{k})} \\
& +\tilde{g} \int_{-\frac{\beta}{2}}^{\frac{\beta}{2}} d \tau \sum_{\alpha \beta} \frac{1}{2} V^{\prime \prime}\left(E_{\alpha \beta}^{\mathrm{sm} .}(\tau)\right) \delta E_{\alpha \beta}^{2}(\tau),
\end{aligned}
$$

where $V^{\prime \prime}\left(E_{\mu}^{\mathrm{sm} .}(\mathbf{r}, \tau)\right)=-4 \pi \delta\left(E_{\mu}^{\mathrm{sm} .}(\mathbf{r}, \tau)-\pi\right)+2=-4 \pi \delta$ $(\tau) \delta_{\mathbf{r}, \mathbf{0}} \delta_{\mu \sigma} / \dot{E}_{\sigma}^{\mathrm{sm} .}(\mathbf{0}, 0)+2$. The normal modes of this action, which include the zero translational mode $\dot{E}^{\text {sm. }}(\mathbf{r}, \tau)$ (see Appendix B for an explicit proof) determine the instanton measure

$$
d \tilde{\xi}_{0} \frac{\sqrt{\left\langle\dot{E}^{\mathrm{sm} \cdot}\right| \dot{E}^{\mathrm{sm} \cdot\rangle}}}{\sqrt{2 \pi}}\left(\frac{\operatorname{det}^{\prime} \tilde{K}}{\operatorname{det} K}\right)^{-\frac{1}{2}}=\frac{\tilde{g} C_{3} d \tilde{\xi}_{0}}{\sqrt{s}}
$$

where $C_{3}=5.13, \tilde{\xi}_{0}$ specifies the position of the instanton core, $\operatorname{det}^{\prime} \tilde{K}$ is the determinant of the kernel of the fluctuation action in Eq. (43), excluding the zero eigenvalue, and det $K$ is the determinant of the kernel of the original action in Eq. (41). See Appendix $\mathrm{C}$ for a detailed derivation of the measure.

\section{Instanton interactions}

The power-law decay (see Appendix A) of the single instanton solution gives rise to long-range interactions between instantons. This infrared effect has consequences for the lowenergy properties of the system. Just like the visons interact via the Coulomb potential, the instantons, which correspond to vison currents, interact in Euclidean space-time via forces that obey the inverse square law.

If the instantons are far apart the interactions will be taking place in a region of space with small electric field. To derive the long-range part of instanton interactions, we can therefore relax the constraint on the electric field $E_{\alpha \beta} \in(-\pi, \pi]$ and integrate it out to obtain an effective action $\mathcal{S}_{\text {inst. }}$ that describes instanton-instanton interactions, i.e., interactions between vison currents,

$$
\mathcal{S}_{\text {inst. }}=\sum_{\tau, \tau^{\prime}} \sum_{\mathbf{r}, \mathbf{r}^{\prime} \in \mathrm{fcc}} V_{\mu \nu}\left(\mathbf{r}-\mathbf{r}^{\prime}, \tau-\tau^{\prime}\right) j_{\mu}(\mathbf{r}, \tau) j_{\nu}\left(\mathbf{r}^{\prime}, \tau^{\prime}\right),
$$

where

$$
\begin{aligned}
V_{\mu \nu}\left(\mathbf{r}-\mathbf{r}^{\prime}, \tau-\tau^{\prime}\right) \\
=\frac{\pi^{2}}{N_{s}} \sum_{\lambda=1,2, \mathbf{k} \in \mathrm{BZ}} \int_{-\infty}^{\infty} \frac{d \omega}{2 \pi} \frac{e^{i \omega\left(\tau^{\prime}-\tau\right)+i \mathbf{k} \cdot\left(\mathbf{r}-\mathbf{r}^{\prime}\right)}}{\frac{\omega^{2}}{4 \tilde{g}}+\frac{z \tilde{g}}{s^{2}} \xi_{\lambda}^{2}(\mathbf{k})} \\
\quad \times U_{\mu \lambda}^{\dagger}(\mathbf{k}) U_{\lambda \nu}(\mathbf{k}) .
\end{aligned}
$$

In the limit where instantons are far apart, it is useful to decompose the sum into its diagonal and off-diagonal parts

$$
\begin{aligned}
\mathcal{S}_{\text {inst. }}= & \sum_{\tau, \tau^{\prime}} \sum_{\mathbf{r}, \mathbf{r}^{\prime} \in \mathrm{fcc}} \tilde{V}_{\mu \nu}\left(\mathbf{r}-\mathbf{r}^{\prime}, \tau-\tau^{\prime}\right) j_{\mu}(\mathbf{r}, \tau) j_{\nu}\left(\mathbf{r}^{\prime}, \tau^{\prime}\right) \\
& +\mu_{\mathrm{I}} \sum_{\tau, \mathbf{r} \in \mathrm{fcc}} j_{\mu}^{2}(\mathbf{r}, \tau),
\end{aligned}
$$

where $\tilde{V}_{\mu \nu}\left(\mathbf{r}-\mathbf{r}^{\prime}, \tau-\tau^{\prime}\right)$ is given by the asymptotic inverse square law of $V_{\mu \nu}\left(\mathbf{r}-\mathbf{r}^{\prime}, \tau-\tau^{\prime}\right)$ with the additional 
constraint that $\tilde{V}_{\mu \mu}(\mathbf{0}, 0)=0$, and the instanton chemical potential (its self-energy) is given by

$$
\begin{aligned}
\mu_{\mathrm{I}}=V_{11}(\mathbf{0}, 0) & =\frac{\pi^{2}}{N_{s}} \sum_{\lambda=1,2, \mathbf{k} \in \mathrm{BZ}} \int_{-\infty}^{\infty} \frac{d \omega}{2 \pi} \frac{U_{1 \lambda}^{\dagger}(\mathbf{k}) U_{\lambda 1}(\mathbf{k})}{\frac{\omega^{2}}{4 \tilde{g}}+\frac{z \tilde{g}}{s^{2}} \xi_{\lambda}^{2}(\mathbf{k})} \\
& =\frac{\pi^{2} s}{\sqrt{z} N_{s}} \sum_{\mathbf{k} \in \mathrm{BZ}} \frac{Z_{11}^{2}(\mathbf{k})}{\left|\xi_{1}(\mathbf{k})\right|^{3}}=C_{2} s
\end{aligned}
$$

where $Z_{11}^{2}(\mathbf{k})$ is given by the matrix product $\sum_{\mu} Z_{1 \mu}(\mathbf{k}) Z_{\mu 1}(\mathbf{k})$ and $C_{2}=0.624$. (Note that the self-energy is exact, i.e., unaffected by the above relaxation of the constraint on the size of the electric field and can be obtained by substituting the single instanton solution in Eq. (38) into the action.) The above decomposition separates the UV (self-energy) and IR (long-range interaction) contributions to the instanton action and can equivalently be obtained by modifying the original action in Eq. (13) as follows. One imposes a small cutoff $\Lambda<<a_{0}$ on the action, i.e., any fluctuations of the gauge fields whose wavelength is not much greater than the lattice spacing are quenched. This has no effect on long-range photon correlations or interactions between instantons or visons that are far apart. It does however give a vanishingly small instanton and vison self-energies, i.e., $\mu_{I} \sim\left(\Lambda / a_{0}\right)^{2} \ll 1$ and $\mu_{V} \sim\left(\Lambda / a_{0}\right) \ll 1$, and so the instanton and vison chemical potential terms need to be added to the action in Eq. (13) to compensate for this. We thus obtain an effective model with a reduced cutoff described by the following action:

$$
\begin{aligned}
\mathcal{S}= & \mathcal{S}_{0}+\int_{-\beta / 2}^{\beta / 2} d \tau \sum_{\gamma}\left[\mu_{V} q_{\gamma}(\tau)^{2}+2 \pi i q_{\gamma}(\tau) \varphi_{\gamma}(\tau)\right] \\
& +\sum_{\tau, \alpha \beta}\left(\mu_{\mathrm{I}} j_{\alpha \beta}(\tau)^{2}+2 \pi i j_{\alpha \beta}(\tau) A_{\alpha \beta}(\tau)\right) \\
& +\sum_{\tau, \gamma} i \theta_{\gamma}(\tau)\left(\Delta_{\tau} q_{\gamma}(\tau)+\operatorname{div}_{\gamma} j(\tau)\right)
\end{aligned}
$$

where

$$
\begin{aligned}
\mathcal{S}_{0}= & \sum_{\omega,|\mathbf{k}|<\Lambda, \lambda=1,2}\left(\frac{\tilde{g} z|\mathbf{k}|^{2} a_{0}^{2}}{s^{2}}+\frac{\omega^{2}}{4 \tilde{g}}\right)\left|A_{\lambda}(\mathbf{k}, \omega)\right|^{2} \\
& +\frac{1}{4 \tilde{g} a_{0}} \sum_{\omega} \int_{|\mathbf{k}|<\Lambda} \frac{d^{3} \mathbf{k}}{(2 \pi)^{3}}|\mathbf{k}|^{2}|\varphi(\mathbf{k}, \omega)|^{2},
\end{aligned}
$$

and the compact Higgs field $\theta_{\gamma}(\tau) \in(-\pi, \pi]$ was introduced to enforce continuity at each tetrahedron site. $\varphi(\mathbf{k}, \tau)$ is the Fourier transform of the coarse-grained scalar potential $\varphi_{\gamma}(\tau)$. The above action is one of the central results of this paper and an effective model for the original action in Eq. (13), valid in the large- $s$ and low-temperature $(\beta \tilde{g} \sqrt{z} / s \gg 1)$ limits.

In the $s \gg 1$ limit, the $j_{\alpha \beta}(\tau)= \pm 1$ instanton configurations dominate in the action in Eq. (49). We can obtain an $\mathrm{O}(2)$ rotor description of visons coupled to the electromagnetic field by summing over these configurations.

$$
\begin{aligned}
Z= & \sum_{q_{\gamma}(\tau)} \int d \theta_{\gamma}(\tau) d \varphi_{\gamma}(\tau) d A_{\alpha \beta}(\tau) e^{-S_{0}} \sum_{n} \frac{e^{-n \mu_{I}}}{n !} \\
& \times e^{\sum_{\gamma} \int_{-\beta / 2}^{\beta / 2} d \tau\left(\mu_{\nu} q_{\gamma}(\tau)^{2}+2 \pi i q_{\gamma}(\tau) \varphi_{\gamma}(\tau)-i q_{\gamma}(\tau) \dot{\theta}_{\gamma}(\tau)\right)}
\end{aligned}
$$

$$
\begin{aligned}
& \times\left(\sum_{\alpha \beta} \int_{-\frac{\beta}{2}}^{\frac{\beta}{2}} \frac{C_{3} \tilde{g} d \tau}{\sqrt{s}}\left(e^{2 \pi i A_{\alpha \beta}+i \Delta_{\alpha \beta} \theta}+e^{-2 \pi i A_{\alpha \beta}-i \Delta_{\alpha \beta} \theta}\right)\right)^{n} \\
\equiv & \sum_{q_{\gamma}(\tau)} \int d \theta_{\gamma}(\tau) d \varphi_{\gamma}(\tau) d A_{\alpha \beta}(\tau) e^{-S_{\mathrm{O}(2)}}
\end{aligned}
$$

where the $\mathrm{O}(2)$ vison action is given by

$$
\begin{aligned}
\mathcal{S}_{\mathrm{O}(2)}= & \mathcal{S}_{0}+\sum_{\gamma} \int_{-\frac{\beta}{2}}^{\frac{\beta}{2}} d \tau\left(\mu_{V} q_{\gamma}(\tau)^{2}+2 \pi i q_{\gamma}(\tau) \varphi_{\gamma}(\tau)\right. \\
& \left.-i q_{\gamma}(\tau) \dot{\theta}_{\gamma}(\tau)\right)-\int_{-\frac{\beta}{2}}^{\frac{\beta}{2}} \frac{C_{3} \tilde{g} e^{-\mu_{I}} d \tau}{\sqrt{s}} \\
& \times \sum_{\alpha \beta} \cos \left(\Delta_{\alpha \beta} \theta(\tau)+2 \pi A_{\alpha \beta}(\tau)\right) .
\end{aligned}
$$

Motivated by the success of our previous work [9], where a large-s expansion was used to obtain the low-energy spectrum for $s=1 / 2$, we believe that $s \rightarrow \infty$ under RG and should be treated as a large parameter. Hence, the last term in the above action should always be thought of as a small perturbation $\left(\tilde{g} e^{-\mu_{I}} / \sqrt{s} \ll \mu_{V}\right)$ and does not drive a condensation of visons (definite phase $\theta_{\gamma}(\tau)$ ) even down to $s=1 / 2$, i.e., QSI is in the deconfined phase for all $s$ values.

\section{GROUND STATE}

At zero temperature vison-antivison pairs will be shortlived virtual excitations. Because of the vison continuity conditions in Eq. (14), they will necessarily be accompanied by instantons forming a loop in Euclidean space-time. The Boltzmann weight of the shortest loop will scale as $e^{-2 \mu_{I}}=$ $e^{-2 C_{2} s}$, and so, in the large $s$ limit, the loops will give a small nonperturbative renormalization of the speed of light and vison Coulomb interaction. There is an energy cost $\left(\mu_{V}\right.$ per unit time) associated with stretching the loop in imaginary time. Loops, where the instantons are close together will therefore dominate. Hence, our calculation of the renormalization of the speed of light presented in Appendix D is not numerically accurate, because the decomposition into UV and IR parts, described in the previous section, only works if the instantons and visons are far apart in Euclidean space-time. At nonzero temperatures, however, we will be considering free visons (rather than vison-antivison pairs), whose instantons will be far apart in the large-s limit and so the UV/IR decomposition will be numerically accurate. (Physical vison excitations live for all imaginary time at nonzero temperature and their instantons can therefore be moved apart in imaginary time with no energy cost.)

Apart from the renormalization of the speed of light, vison-antivison pairs, will also make another contribution to the $S^{z}$ correlation (which is proportional to the neutron scattering intensity) that scales as $e^{-2 \mu_{I}}=e^{-2 C_{2} s}$. Generation of vison-antivison pairs by passing neutrons will give rise to a continuum of excitations above an energy equal to $2 \mu_{V}$. Figure 1 shows this vison continuum above the linearly dispersing photon excitation, as would be seen in a typical neutron scattering experiment. See Appendix D for a detailed calculation of the continuum. Note that the presence of the 

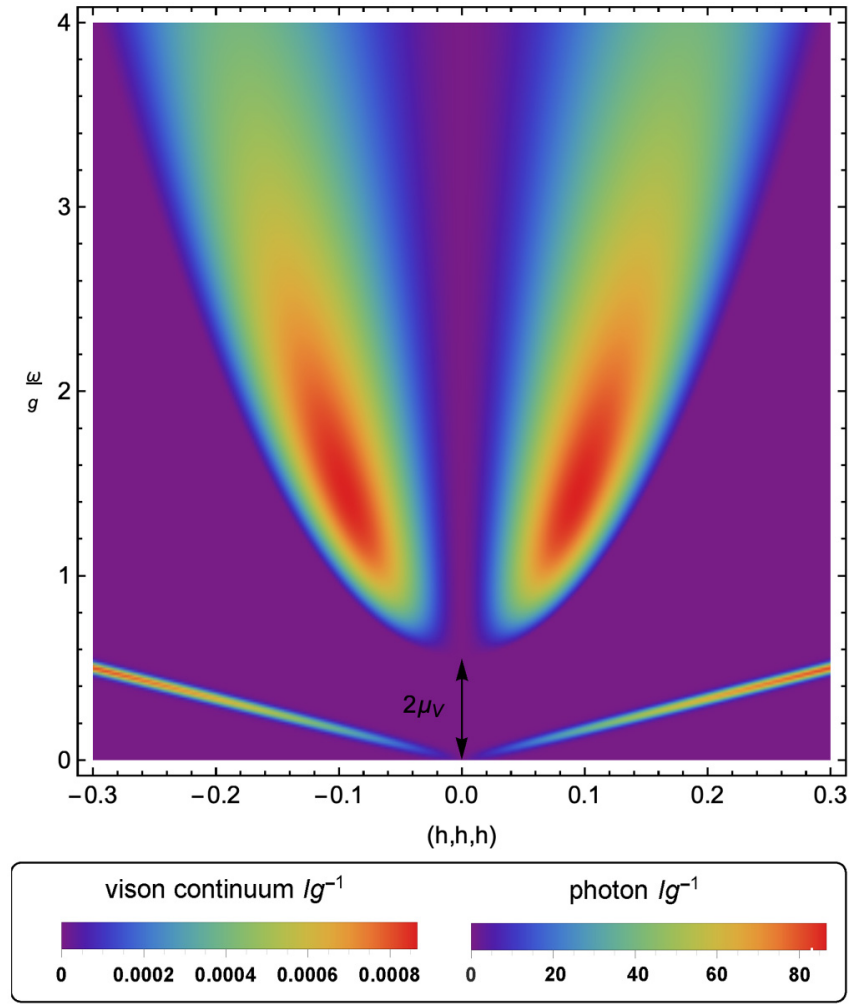

FIG. 1. The unpolarized scattering intensity at $T=0$ for $s=\frac{1}{2}$, as might be seen in a typical neutron diffraction experiment along the direction $\mathbf{k}=\frac{2 \pi}{a_{0}}(h, h, h)$. Generation of vison-antivison pairs gives rise to a continuum of excitations above $2 \mu_{V}$. Below, we can see the linearly dispersing photon excitation. To simulate the finite resolution of the measuring apparatus, we have convoluted the photon intensity with a Gaussian of width $0.02 \mathrm{~g}$. (Note that the photon and vison spectra have separate intensity scales and the relative intensity will be very sensitive to the resolution of the measuring apparatus.)

vison continuum had been expected in previous works, e.g., Ref. [13]. In this work, our focus will instead be on the effect of thermally excited visons on the photon part of the scattering spectrum.

\section{NONZERO TEMPERATURES}

\section{A. 3D plasma of visons}

At nonzero temperatures the visons are not so innocuous. They become real thermal excitations and form a 3D Coulomb gas. The gas is always in the plasma phase [30], however large the vison self-energy $\mu_{V}$. To see this, we consider the RG flow equations of the gas parameters. In the $s \rightarrow \infty$ limit, where the visons are static, and at low temperatures, where $\beta \mu_{V} \gg 1$, the $\mathrm{RG}$ equations are given by [30]

$$
\begin{gathered}
\frac{d K^{-1}}{d l}=K^{-1}+y^{2}, \\
\frac{d y}{d l}=3 y-K y,
\end{gathered}
$$

where the fugacity $y \equiv e^{-\beta \mu_{V}}$ and $K$ is the coefficient of the vison Coulomb interaction energy $\tilde{V}(\mathbf{r})$ in Eq. (35) multiplied by $\beta$. Under RG, $\beta \mu_{V}$ grows linearly from its initial bare value of $\sim \beta \tilde{g}$, whereas $K$ decays exponentially from its initial bare value of $\sim \beta \tilde{g}$. Eventually, once $K$ has decayed sufficiently, $\beta \mu_{V}$ begins to fall linearly. Hence, the visons dissociate before they begin to proliferate. Even though initially the vison self-energy and interaction energy are comparable, at long length scales, the interaction can be neglected by comparison with the self-energy and the visons can be treated as free particles. Classical Monte Carlo calculations of Ref. [26] found that the energy of a nearest-neighbor vison pair is actually lower than that of a single vison, so that at low temperatures, vison pairs are the dominant species and the visons form a weak electrolyte. Here, we argue that the model can be mapped, by integrating out tightly bound vison pairs, to a coarse-grained 3D Coulomb gas where free visons are the dominant species. Note that a finite vison inertia (finite $s$ ) should only increase their ability to screen each other, reducing interactions even further. Therefore, if the visons are in the plasma phase for infinite $s$, we expect them to also be in the plasma phase for finite $s$

\section{B. Semiclassical limit at low temperatures}

We now consider the dynamics of the vison plasma at small nonzero temperatures. At low temperatures the typical vison spacing will be large and their hardcore interactions negligible. We can therefore treat visons as free bosons of two flavors coupled to the electromagnetic field. Introducing two complex bosonic fields $\Psi_{\gamma}^{+}(\tau)$ and $\Psi_{\gamma}^{-}(\tau)$ corresponding to visons and antivisons respectively, the vison plasma action can be written as follows:

$$
\begin{aligned}
\mathcal{S}_{\mathrm{VP}}\left[\Psi_{\gamma}^{\sigma}(\tau)\right] & =\mathcal{S}_{0}+\sum_{\sigma= \pm} \int_{-\frac{\beta}{2}}^{\frac{\beta}{2}} d \tau\left[\sum_{\gamma} \bar{\Psi}_{\gamma}^{\sigma}\left(\partial_{\tau}+2 \pi i \sigma \varphi_{\gamma}+\mu_{V}\right) \Psi_{\gamma}^{\sigma}\right. \\
& \left.-\frac{C_{3} \tilde{g} e^{-\mu_{\mathrm{I}}}}{\sqrt{s}} \sum_{\alpha \beta}\left(\bar{\Psi}_{\alpha}^{\sigma} \Psi_{\beta}^{\sigma} e^{-2 \pi i \sigma A_{\alpha \beta}}+\text { H.c }\right)\right]
\end{aligned}
$$

which is the action of lattice bosons coupled to a gauge field. Notice that the above action is particle conserving, i.e., visonantivison pair creation and annihilation has been neglected. As demonstrated in Appendix D, these can be treated perturbatively in the large-s limit and give rise to a continuum of excitations above $2 \mu_{V}$ and a small renormalization of the speed of light. Our focus here will be on a more dramatic reshaping of the photon spectrum. At sufficiently low temperatures, where $\beta \tilde{g} e^{-\mu_{\mathrm{I}}} / \sqrt{s} \gg 1$, [31] the visons move at the bottom of the band and a continuum (low $\mathbf{k}$ ) approximation for the bosonic fields can be made

$$
\begin{aligned}
\mathcal{S}_{\mathrm{VP}}\left[\Psi^{\sigma}(\mathbf{r}, \tau)\right]= & \mathcal{S}_{0}+\sum_{\sigma= \pm} \int_{-\frac{\beta}{2}}^{\frac{\beta}{2}} d \tau \int \frac{d^{3} \mathbf{r}}{\left(a_{0}^{3} / 8\right)}\left[\mu_{V}\left|\Psi^{\sigma}\right|^{2}\right. \\
& +\frac{8 \tilde{g} C_{3} a_{0}^{2} e^{-\mu_{\mathrm{I}}}}{\sqrt{s}}\left|\nabla \Psi^{\sigma}-2 \pi i \sigma \mathbf{A} \Psi^{\sigma}\right|^{2} \\
& \left.+\bar{\Psi}^{\sigma}\left(\partial_{\tau}+2 \pi i \sigma \varphi\right) \Psi^{\sigma}\right]
\end{aligned}
$$


where $\mathbf{A}(\mathbf{r}, \tau)$ [defined by $A^{\mu}(\mathbf{r}, \tau)=\mathbf{A}(\mathbf{r}, \tau) \cdot \mathbf{e}_{\mu}$ ] and $\varphi(\mathbf{r}, \tau)$ are coarse-grained vector and scalar potentials. Note that we have neglected nonperturbative corrections to the vison energy $\mu_{V}$ of order $e^{-C_{2} s}$. This is for self-consistency as we have already neglected perturbative corrections which come from higher order terms in the expansion of the Hamiltonian in Eq. (12). The vison plasma action describes bosons with a thermal activation energy of $\mu_{V}$, effective mass of $m^{*}=\frac{\hbar^{2} \sqrt{s} \mu^{\mu_{I}}}{16 C_{3} a_{0}^{2} \tilde{g}}$ and an effective charge of $2 \pi \hbar$.

The bosonic excitations can be treated semiclassically at low temperatures because their typical separation $\sim a_{0} e^{\beta \mu_{V}} \gg$ $\lambda_{\mathrm{B}}$, where $\lambda_{\mathrm{B}}=\sqrt{\frac{2 \pi \hbar^{2}}{k T m^{*}}}$ is the thermal de Broglie wavelength of the bosons. The vison excitations are thus not quantum degenerate and can be treated through a single-particle formalism. Considering the Feynman path integral of a single vison excitation, we obtain

$$
\begin{aligned}
S_{\text {single }}=\int_{-\frac{\beta}{2}}^{\frac{\beta}{2}} d \tau( & \frac{m^{*}}{2 \hbar^{2}}\left(\partial_{\tau} \mathbf{x}\right)^{2} \pm 2 \pi i \mathbf{A}(\mathbf{x}, \tau) \cdot \partial_{\tau} \mathbf{x} \\
& \pm 2 \pi i \varphi(\mathbf{x}, \tau))
\end{aligned}
$$

where $\mathbf{x}(\tau)$ is the vison's position vector and \pm depends on whether the vison is positively or negatively charged. The typical quantum fluctuations in the position of the particle will be

$$
\langle|\Delta \mathbf{x}|\rangle \sim \sqrt{2 \pi} \beta\left\langle\left|\partial_{\tau} \mathbf{x}\right|\right\rangle=\sqrt{\frac{2 \pi \hbar^{2} \beta}{m^{*}}} \equiv \lambda_{B} .
$$

At low temperatures, fractional variations in $\mathbf{A}(\mathbf{x}, \tau)$, or $\varphi(\mathbf{x}, \tau)$, due to the fluctuating position of the particle $\mathbf{x}$ will be negligible

$$
\frac{|\nabla \mathbf{A}|}{|\mathbf{A}|} \lambda_{B} \sim \frac{\lambda_{B}}{\lambda_{A}} \sim \sqrt{\frac{1}{2 \pi \beta m^{*} c^{2}}} \ll 1,
$$

where $\lambda_{A}=2 \pi \hbar c \beta$ is the typical wavelength of the gauge field and $c=\frac{2 \tilde{g} \sqrt{z} a_{0}}{s \hbar}$ is the speed of the photon excitation. The temperatures here are small relative to the vison rest energy $m^{*} c^{2} \sim \tilde{g} e^{\mu_{I}}$. At these temperatures, the visons are moving nonrelativistically with a typical speed $v=\left(\beta m^{*}\right)^{-\frac{1}{2}} \ll c$. We can therefore approximate

$$
\begin{aligned}
& \mathbf{A}(\mathbf{x}, \tau) \approx \mathbf{A}(\langle\mathbf{x}\rangle, \tau), \\
& \varphi(\mathbf{x}, \tau) \approx \varphi(\langle\mathbf{x}\rangle, \tau),
\end{aligned}
$$

where $\langle\mathbf{x}\rangle$ is the average position of the vison. With regards to vison dynamics, we have neglected the Lorentz force experienced by visons by making the above approximation, only leaving the electric field force. This is of course a good approximation at nonrelativistic speeds. The particle's position can be decomposed as

$$
\mathbf{x}(\tau)=\langle\mathbf{x}\rangle+\frac{1}{\sqrt{\beta}} \sum_{\omega \neq 0} \mathbf{x}(\omega) e^{i \omega \tau},
$$

where the Matsubara frequency $\omega=\frac{2 \pi n}{\beta}$ and $n$ is an integer. Using the above approximation for the vector and scalar potentials, the single-particle vison action becomes

$$
\begin{aligned}
S_{\text {single }}= & \sum_{\omega \neq 0}\left(\frac{m^{*} \omega^{2}}{2 \hbar^{2}}|\mathbf{x}(\omega)|^{2} \pm 2 \pi \omega \mathbf{A}(\langle\mathbf{x}\rangle,-\omega) \cdot \mathbf{x}(\omega)\right) \\
& \pm 2 \pi i \sqrt{\beta} \varphi(\langle\mathbf{x}\rangle, \omega=0) .
\end{aligned}
$$

We note here that visons do not couple to the static component of the gauge field $\mathbf{A}(\mathbf{x}, \omega=0)$, and hence also the static background field $A_{\alpha \beta}^{0}$, be it from the half-integer constraint on the magnetic field or from magnetic monopoles introduced into the system. This is because we have neglected the Lorentz force experienced by visons. The visons are only coupled to the electric field in our description which can only be induced by a time-varying magnetic field. Of course, visons also respond to static magnetic fields. Loops of vison currents will align in the direction of an applied static magnetic field through the action of the Lorentz force. This effect will generate $\mathrm{a}(\boldsymbol{\nabla} \times \mathbf{A})^{2}$ term in the action and only renormalize the speed of light slightly. The effect we are describing here is far more dramatic. The response of visons to the electromotive force induced by a time varying magnetic field, as we shall see shortly, generates a mass gap in the photon spectrum.

Integrating over the fluctuations in the particle's position $\mathbf{x}(\omega)$ with $\omega \neq 0$, we obtain

$$
S_{\text {single }}=\frac{2 \pi^{2} \hbar^{2}}{m^{*}} \sum_{\omega \neq 0}|\mathbf{A}(\langle\mathbf{x}\rangle, \omega)|^{2} \pm 2 \pi i \sqrt{\beta} \varphi(\langle\mathbf{x}\rangle, \omega=0) .
$$

Summing over many-particle configurations, we obtain the partition function of the system

$$
\begin{aligned}
Z= & \prod_{\gamma, \alpha \beta} \int d \varphi_{\gamma}(\tau) d A_{\alpha \beta}(\tau) e^{-S_{0}} \sum_{n} \frac{e^{-n \beta \mu_{V}}}{n !} \\
& \times \prod_{i=1}^{n} \int \frac{d^{3} \mathbf{x}_{i}}{\lambda_{B}^{3}} e^{-\frac{2 \pi^{2} \hbar^{2}}{m^{*}} \sum_{\omega \neq 0}\left|\mathbf{A}\left(\mathbf{x}_{i}, \omega\right)\right|^{2}} \\
& \times\left(e^{2 \pi i \sqrt{\beta} \varphi\left(\mathbf{x}_{i}, 0\right)}+e^{-2 \pi i \sqrt{\beta} \varphi\left(\mathbf{x}_{i}, 0\right)}\right) \\
= & \prod_{\gamma, \alpha \beta} \int d \varphi_{\gamma}(\tau) d A_{\alpha \beta}(\tau) \exp \left[-S_{0}+\int \frac{d^{3} \mathbf{x}}{\lambda_{B}^{3}} e^{-\beta \mu_{V}}\right. \\
& \left.\times e^{-\frac{2 \pi^{2} \hbar^{2}}{m^{*}} \sum_{\omega \neq 0}|\mathbf{A}(\mathbf{x}, \omega)|^{2}} 2 \cos (2 \pi \sqrt{\beta} \varphi(\mathbf{x}, 0))\right]
\end{aligned}
$$

At low temperatures, we are in the Debye limit $e^{-\beta \mu_{V}} \ll 1$, where the action can be expanded to quadratic order. One can see that the gauge fields are effectively small in this limit by performing the following rescaling:

$$
\begin{aligned}
\mathbf{x} & \rightarrow e^{\beta \mu_{V} / 3} \mathbf{x}, \\
\omega & \rightarrow e^{-\beta \mu_{V} / 3} \omega, \\
\mathbf{A}(\mathbf{x}, \omega) & \rightarrow e^{-\beta \mu_{V} / 6} \mathbf{A}(\mathbf{x}, \omega), \\
\varphi(\mathbf{x}, \omega) & \rightarrow e^{-\beta \mu_{V} / 6} \varphi(\mathbf{x}, \omega) .
\end{aligned}
$$




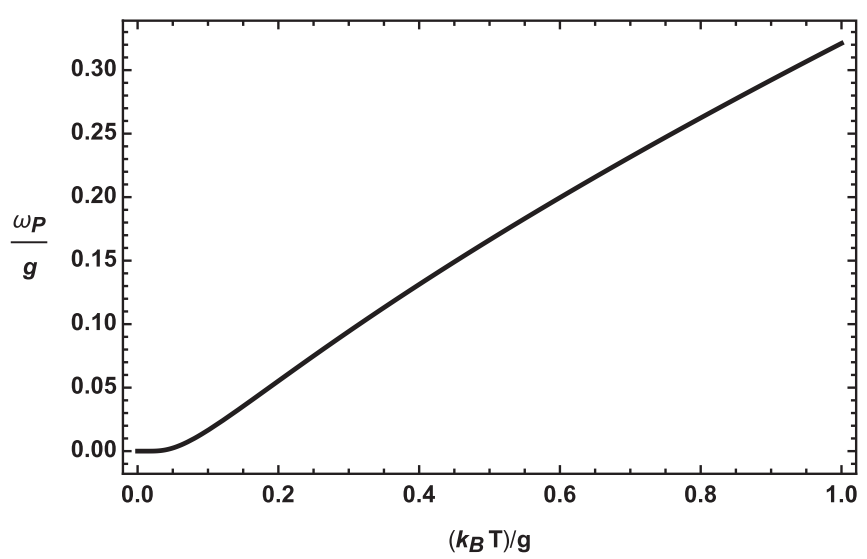

FIG. 2. Plasma frequency as a function of temperature. At higher temperatures the plasma frequency will level off as the vison density saturates.

To quadratic order, the gauge part of the action is given by

$$
\begin{aligned}
S_{\text {gauge }}= & \sum_{\omega,|\mathbf{k}|<\Lambda, \lambda=1,2}\left(\frac{\tilde{g} z a_{0}^{2}|\mathbf{k}|^{2}}{s^{2}}+\frac{\omega^{2}}{4 \tilde{g}}+\left(1-\delta_{\omega, 0}\right) \frac{\omega_{p}^{2}}{4 \tilde{g}}\right) \\
& \times\left|A_{\lambda}(\mathbf{k}, \omega)\right|^{2}+\sum_{\omega} \int_{|\mathbf{k}|<\Lambda} \frac{d^{3} \mathbf{k}}{(2 \pi)^{3}} \frac{1}{4 \tilde{g} a_{0}} \\
& \times\left(|\mathbf{k}|^{2}+\lambda_{D}^{-2} \delta_{\omega, 0}\right)|\varphi(\mathbf{k}, \omega)|^{2},
\end{aligned}
$$

where the Debye length is given by

$$
\lambda_{\mathrm{D}}=\left(16 \pi^{2} a_{0} \lambda_{B}^{-3} \beta \tilde{g} e^{-\beta \mu_{V}}\right)^{-\frac{1}{2}},
$$

and in the Debye limit $e^{\beta \mu_{V}} \gg 1$, there are many visons $e^{-\beta \mu_{V}}\left(\lambda_{\mathrm{D}} / \lambda_{B}\right)^{3} \sim e^{\beta \mu_{V} / 2} \gg 1$ in the Debye volume. The plasma frequency (multiplied by $\hbar$ ) is given by

$$
\omega_{p}=\sqrt{\frac{16 \tilde{g} \pi^{2} \hbar^{2} a_{0} e^{-\beta \mu_{V}}}{\lambda_{B}^{3} m^{*}}},
$$

and agrees with the continuum derivation presented in Appendix G. The vison dynamics has generated a dynamical mass gap for the gauge field, equal to the plasma frequency. Figure 2 shows the behavior of the plasma frequency with temperature. The mass gap is dynamical because it does not couple to the static $\omega=0$ component of the gauge field. We have derived the mass gap in the large- $s$ and low-temperature limits, where $s \gg 1, \beta \tilde{g} \sqrt{z} / s \gg 1$ (which implies $\beta \mu_{V} \gg 1$ ), and $\beta \tilde{g} e^{-\mu_{I}} / \sqrt{s} \gg 1$, i.e., the temperature is small by comparison with the photon and vison bandwidths respectively. However, we expect the above picture to extend to moderate temperatures too, although the instanton energy, and hence the mass gap, would acquire significant renormalization due to finite-size effects along imaginary time. Note that we have also not included tightly bound vison pairs in our semiclassical analysis of vison dynamics since their oscillations do not contribute to the generation of the mass gap [32] but only serve to renormalize the speed of light (see Appendix D for a detailed calculation of this effect).

\section{Inelastic magnetic response}

We can now extract several correlators from the above quadratic gauge action. In particular, relevant for neutron scattering experiments are magnetic field correlators:

$$
\begin{aligned}
\chi_{\lambda}(\mathbf{k}, i \omega) & \equiv\left\langle\left|S_{\lambda}^{z}(\mathbf{k}, \omega)\right|^{2}\right\rangle \\
& =\frac{2 \tilde{g} \xi_{\lambda}^{2}(\mathbf{k})}{-(i \omega)^{2}+E_{\mathbf{k}}^{2}}+\delta_{\omega, 0} C_{\lambda}(\mathbf{k}),
\end{aligned}
$$

where $\quad E_{\mathbf{k}}=\sqrt{4 \tilde{g}^{2} z \xi_{1}^{2}(\mathbf{k}) / s^{2}+\omega_{p}^{2}} \quad$ and $\quad C_{\lambda}(\mathbf{k})=\frac{2 \tilde{g} \xi_{\lambda}^{2}(\mathbf{k})}{E_{\mathbf{k}}^{2}-\omega_{p}^{2}}-$ $\frac{2 \tilde{g} \xi_{\zeta^{2}}^{2}(\mathbf{k})}{E_{\mathbf{k}}^{2}}$. (Notice that we have replaced the low-k expansion with $\xi_{\lambda}(\mathbf{k})$ to restore periodicity across the Brillouin zone.)

\section{Equal-time structure factor}

A particularly useful quantity to measure in neutron diffraction experiments is the equal-time (energy integrated) structure factor. We calculate the structure factor in the spinflip channel for a polarized neutron-scattering experiment considered by Ref. [33],

$$
\begin{aligned}
S^{y y}(\mathbf{k}) \equiv & \sum_{\mu, v}\left\langle S_{\mu}^{z}(\mathbf{k}, \tau=0) S_{\nu}^{z}(-\mathbf{k}, \tau=0)\right\rangle\left(\hat{\mathbf{e}}_{\mu} \cdot \hat{\mathbf{e}}_{y}\right)\left(\hat{\mathbf{e}}_{v} \cdot \hat{\mathbf{e}}_{y}\right) \\
= & \frac{1}{\beta} \sum_{\mu, v} \sum_{\omega, \lambda=1,2} \chi_{\lambda}(\mathbf{k}, i \omega)\left(\hat{\mathbf{e}}_{\mu} \cdot \hat{\mathbf{e}}_{y}\right)\left(\hat{\mathbf{e}}_{v} \cdot \hat{\mathbf{e}}_{y}\right) U_{\mu \lambda}^{\dagger}(\mathbf{k}) \\
& \times U_{\nu \lambda}^{\dagger}(-\mathbf{k}) \\
= & \tilde{g} \sum_{\mu \nu} Z_{\mu \nu}^{2}(\mathbf{k})\left(\hat{\mathbf{e}}_{\mu} \cdot \hat{\mathbf{e}}_{y}\right)\left(\hat{\mathbf{e}}_{v} \cdot \hat{\mathbf{e}}_{y}\right) \\
& \times\left(\frac{1+2 n_{B}\left(E_{\mathbf{k}}\right)}{E_{\mathbf{k}}}+\frac{T C_{1}(\mathbf{k})}{\tilde{g} \xi_{1}^{2}(\mathbf{k})}\right)
\end{aligned}
$$

where $\hat{\mathbf{e}}_{y}=\frac{\mathbf{k} \times(1,-1,0)}{|\mathbf{k} \times(1,-1,0)|}, n_{B}\left(E_{\mathbf{k}}\right)=\frac{1}{e^{\beta \mathbf{k}_{\mathbf{k}}-1}}$, and $Z_{\mu \nu}^{2}(\mathbf{k})$ is given by the matrix product $\sum_{\sigma} Z_{\mu \sigma}(\mathbf{k}) Z_{\sigma \nu}(\mathbf{k})$.

Because the mass gap $\omega_{p}$ does not couple to the zero Matsubara frequency component, it can only have an effect on the equal-time structure factor at low temperatures, but this is precisely where it is exponentially damped. The effects of the mass gap on the equal-time structure factor are therefore small across the temperature range and cannot be easily seen as shown by Figs. 3 and 4. Motivated by the success of our previous work [9], we extrapolated the large-s results to $s=\frac{1}{2}$. Both figures are in good agreement with the results of Monte Carlo simulations of Ref. [14].

\section{The dynamical structure factor}

We should instead look for experimental signatures of the mass gap in the unintegrated neutron scattering spectra. We do this by calculating the dynamical structure factor

$$
S_{\lambda}(\mathbf{k}, \omega)=\int d t\left\langle\hat{S}_{\lambda}^{z}(\mathbf{k}, t) \hat{S}_{\lambda}^{z}(-\mathbf{k}, 0)\right\rangle e^{i \omega t} .
$$




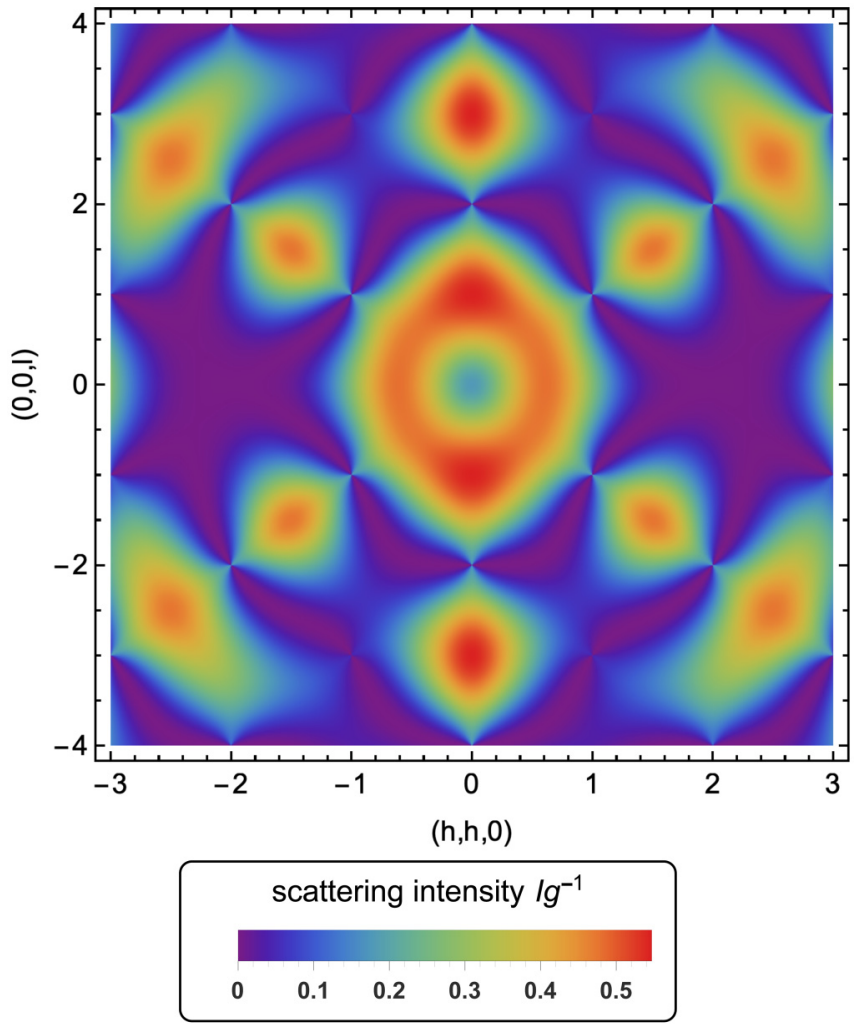

FIG. 3. Equal-time structure factor in the spin-flip channel along $\mathbf{k}=\frac{2 \pi}{a_{0}}(h, h, l)$ at $T=0.15 g$, for $s=\frac{1}{2}$ and with the vison contribution.

We can analytically continue the analytic part of $\chi(\mathbf{k}, i \omega)$ to obtain the spectral density

$$
\begin{aligned}
\rho_{\lambda}(\mathbf{k}, \omega) & =\operatorname{Im} \lim _{\epsilon \rightarrow 0} \chi_{\lambda}(\mathbf{k}, \omega+i \epsilon) \\
& =\frac{\tilde{g} \pi \xi_{\lambda}^{2}(\mathbf{k})}{E_{\mathbf{k}}}\left(\delta\left(\omega-E_{\mathbf{k}}\right)-\delta\left(\omega+E_{\mathbf{k}}\right)\right) .
\end{aligned}
$$

The structure factor is then given by

$$
\begin{aligned}
S_{\lambda}(\mathbf{k}, \omega)= & 2 \pi T C_{\lambda}(\mathbf{k}) \delta(\omega)+\frac{2 \rho_{\lambda}(\mathbf{k}, \omega)}{1-e^{-\beta \omega}} \\
= & \frac{2 \tilde{g} \pi \xi_{\lambda}^{2}(\mathbf{k})}{E_{\mathbf{k}}}\left(n_{B}\left(E_{\mathbf{k}}\right) \delta\left(\omega+E_{\mathbf{k}}\right)+\left(1+n_{B}\left(E_{\mathbf{k}}\right)\right)\right. \\
& \left.\times \delta\left(\omega-E_{\mathbf{k}}\right)\right) .+2 \pi T C_{\lambda}(\mathbf{k}) \delta(\omega)
\end{aligned}
$$

See Ref. [15] for further details of the relations between $\chi_{\lambda}(\mathbf{k}, \omega), S_{\lambda}(\mathbf{k}, \omega)$, and $\rho_{\lambda}(\mathbf{k}, \omega)$. The presence of the delta function in the dynamical structure factor signals that correlations of the magnetic field are infinitely long-lived in time. This is because of persistent vison currents that are excited (through electromagnetic induction) with any fluctuation of the magnetic field. These currents and the magnetic field they give rise to do not decay with time.

The total unpolarized scattering intensity is proportional to [14]

$$
I(\mathbf{k}, \omega)=\sum_{\mu \nu}\left(\hat{\mathbf{e}}_{\mu} \cdot \hat{\mathbf{e}}_{v}-\frac{\left(\mathbf{k} \cdot \hat{\mathbf{e}}_{\mu}\right)\left(\mathbf{k} \cdot \hat{\mathbf{e}}_{v}\right)}{\mathbf{k}^{2}}\right) S^{\mu v}(\mathbf{k}, \omega),
$$

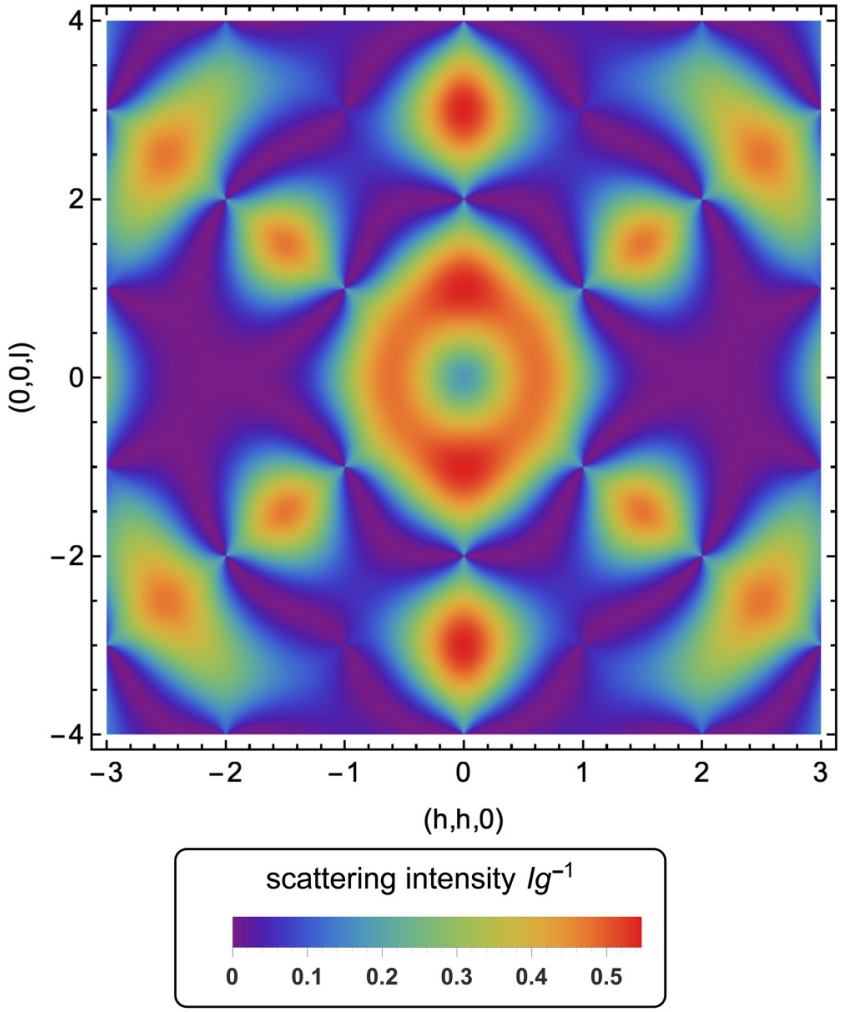

FIG. 4. Equal-time structure factor in the spin-flip channel along $\mathbf{k}=\frac{2 \pi}{a_{0}}(h, h, l)$ at $T=0.15 g$, for $s=\frac{1}{2}$ and without the vison contribution (i.e., with $\omega_{p}$ set to zero). Figures 3 and 4 look identical to the eye and there are no clear signatures of visons.

where $S^{\mu \nu}(\mathbf{k}, \omega)=\sum_{\lambda=1,2} S_{\lambda}(\mathbf{k}, \omega) U_{\mu \lambda}^{\dagger}(\mathbf{k}) U_{\nu \lambda}^{\dagger}(-\mathbf{k})$. The results are plotted in Figs. 5 and 6 and show a clear development of a mass gap at nonzero temperatures, that are relatively small with respect to the photon bandwidth for $s=\frac{1}{2}$.

We compare our dynamical structure factor plots with the quantum Monte Carlo calculations of Ref. [20]. The reported bandwidth $(4.28 g)$ is significantly higher than the one we calculate $(0.6 g)$. However, we believe this discrepancy is mostly due to higher order corrections in the $1 / s$ expansion. In fact, our previous work [9] shows that the next order already renormalizes the bandwidth from $0.6 \mathrm{~g}$ to $1.6 \mathrm{~g}$. Further, the plasma frequency and the speed of light should not be compared numerically in our calculation. This is because the calculation of the former is nonperturbative and includes all orders in $1 / s$, whereas the speed of light has only been calculated to finite order in $1 / s$. This does not impact the main observation though, which is that the photon acquires a mass gap equal to the plasma frequency. Our dynamical structure plots are in rough qualitative agreement with the work of Ref. [20]. However, the quantum Monte Carlo calculations do not have the required resolution to ascertain whether the photon dispersion is linear, let alone whether there is a small energy gap.

\section{Susceptibility}

As discussed previously in this section, the mass gap is a consequence of the induced electric field coupling to the 


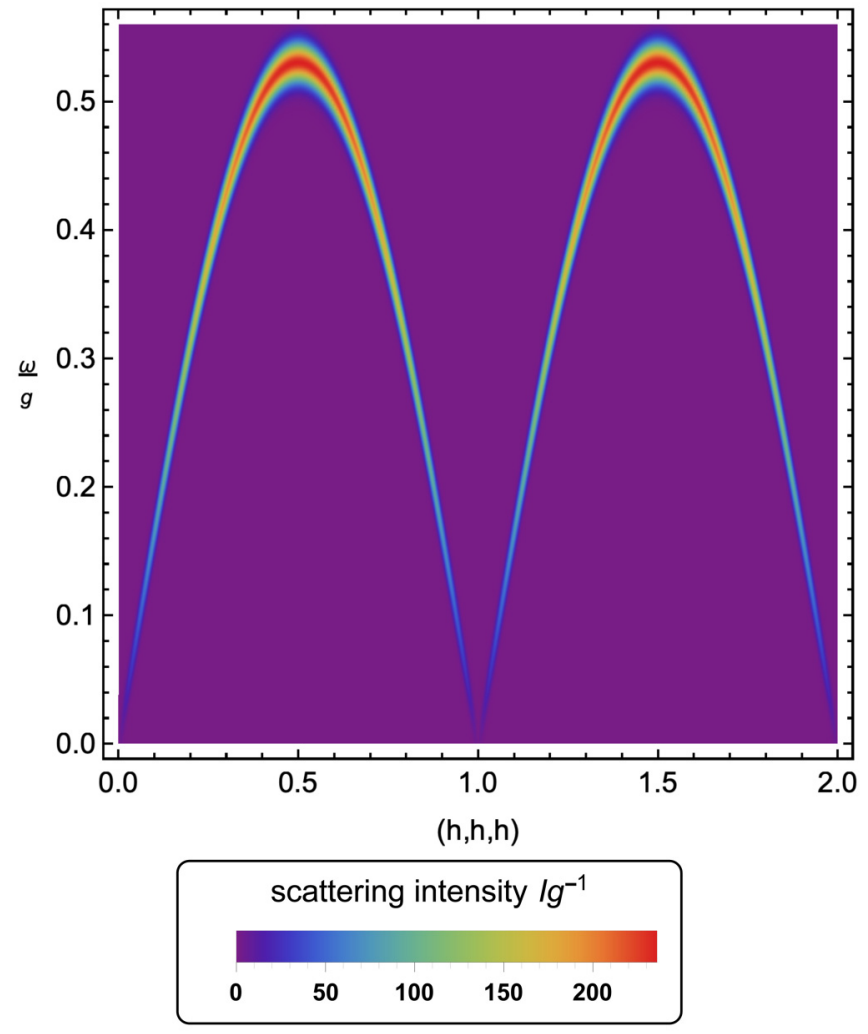

FIG. 5. The unpolarized scattering intensity at $T=0$ for $s=\frac{1}{2}$ with the vison contribution, as might be seen in a typical neutron diffraction experiment along the direction $\mathbf{k}=\frac{2 \pi}{a_{0}}(h, h, h)$. To simulate the finite resolution of the measuring apparatus, we have convoluted the intensity with a Gaussian of width $0.01 \mathrm{~g}$.

visons. Hence, it only shows up in the presence of timevarying magnetic fields. To demonstrate this, we calculate here the static isothermal and isolated susceptibilities using the formulas derived in Ref. [15]. For the isothermal susceptibility, we assume the system to be in thermal equilibrium at temperature $T$ and in the presence of the following time-independent perturbation in the Hamiltonian $\delta \hat{H}=$ $-\frac{h}{2}\left(\hat{S}_{\lambda}^{z}(\mathbf{k})+\hat{S}_{\lambda}^{z}(-\mathbf{k})\right)$. The isothermal susceptibility is then defined as

$$
\begin{aligned}
\chi_{\lambda}^{T}(\mathbf{k}) & \equiv \frac{1}{2} \frac{\delta\left\langle\hat{S}_{\lambda}^{z}(\mathbf{k})+\hat{S}_{\lambda}^{z}(-\mathbf{k})\right\rangle_{T}}{\delta h} \\
& =\int \frac{d \omega}{2 \pi} \frac{\rho_{\lambda}(\mathbf{k}, \omega)}{\omega}+\frac{\beta C_{\lambda}(\mathbf{k})}{2} \\
& =\frac{\tilde{g} \xi_{\lambda}^{2}(\mathbf{k})}{E_{\mathbf{k}}^{2}-\omega_{p}^{2}}=\frac{1}{4 g s^{4} z} \text { for } \lambda=1 \text { and } 2,
\end{aligned}
$$

where the expectation value is taken with respect to the thermal ensemble. We can see that the isothermal transverse ( $\lambda=1$ and 2) susceptibility is a constant, and as expected, is unaffected by visons because the applied magnetic field is time-independent. We would obtain the same expression for a massless photon. (Note that including in our description the effects of the Lorentz force on visons, from the timeindependent magnetic field, would simply lead to a renormal-

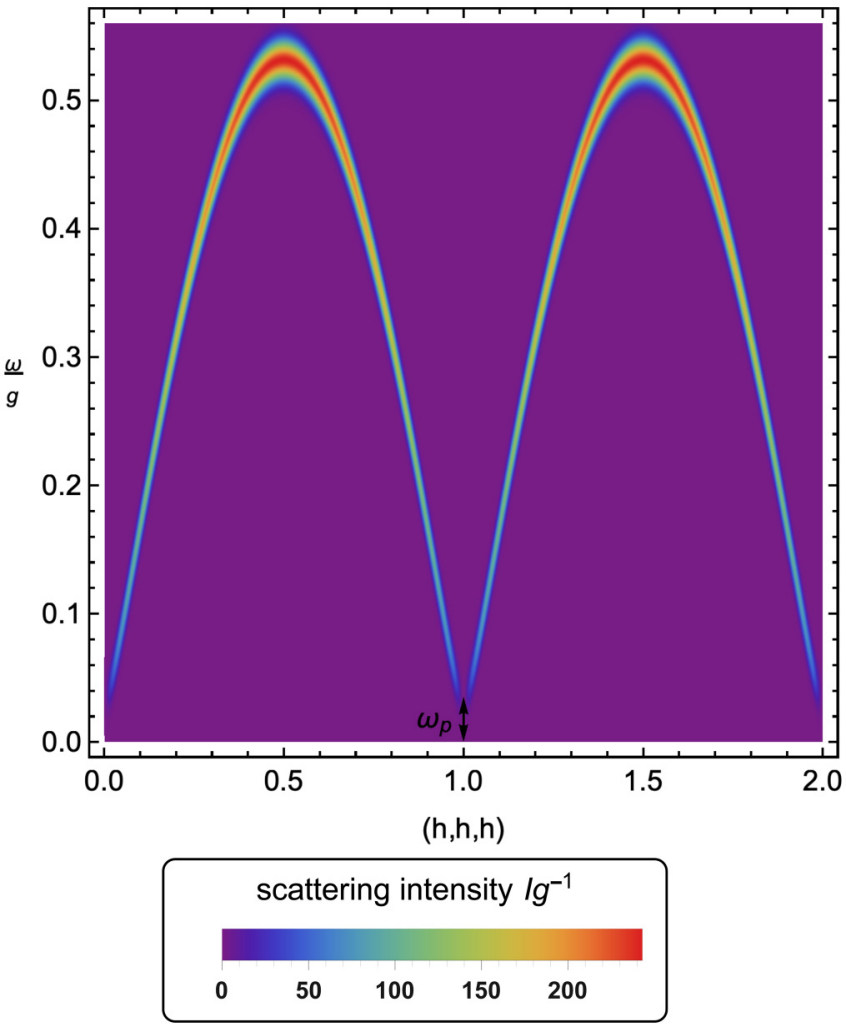

FIG. 6. The unpolarized scattering intensity at $T=0.15 \mathrm{~g}$ for $s=\frac{1}{2}$ with the vison contribution, as might be seen in a typical neutron diffraction experiment along the direction $\mathbf{k}=\frac{2 \pi}{a_{0}}(h, h, h)$. Notice the development of a sizable gap equal to $\omega_{p}$ at temperatures that are relatively small by comparison with the photon bandwidth. To simulate the finite resolution of the measuring apparatus, we have convoluted the intensity with a Gaussian of width $0.01 \mathrm{~g}$.

ization of the speed of the photon excitation and only change the value of the above constant.)

For the isolated susceptibility, we assume the system to be isolated at all times and in thermal equilibrium to begin with i.e., at $t \rightarrow-\infty$. A time-dependent perturbation is then slowly turned on $\delta \hat{H}(t)=-\frac{h}{2}(1-$ $\Theta(t)) e^{\epsilon t}\left(\hat{S}_{\lambda}^{z}(\mathbf{k})+\hat{S}_{\lambda}^{z}(-\mathbf{k})\right)$, where $\Theta(t)$ is the Heaviside step function and $\epsilon \rightarrow 0^{+}$. The isolated susceptibility is then defined as

$$
\begin{aligned}
\chi_{\lambda}^{I}(\mathbf{k}) & \equiv \frac{1}{2} \frac{\delta\left\langle\hat{S}_{\lambda}^{z}(\mathbf{k})+\hat{S}_{\lambda}^{z}(-\mathbf{k})\right\rangle_{I}}{\delta h}=\int \frac{d \omega}{2 \pi} \frac{\rho_{\lambda}(\mathbf{k}, \omega)}{\omega} \\
& =\frac{\tilde{g} \xi_{\lambda}^{2}(\mathbf{k})}{E_{\mathbf{k}}^{2}}=\frac{1}{4 g s^{4}} \frac{1}{1+\left(\frac{\omega_{p} a_{0}}{\hbar c \xi_{1}(\mathbf{k})}\right)^{2}} \quad \text { for } \lambda=1 \text { and } 2,
\end{aligned}
$$

where the expectation value is evaluated at $t=0$ for the isolated system. We can see that the isolated transverse $(\lambda=$ 1 and 2) susceptibility is no longer a constant but begins to fall to zero at small $|\mathbf{k}| \sim \omega_{p} /(\hbar c)$ (see Fig. 7). This is due to the screening contribution of persistent vison currents that are set up as the external field is switched on, and is analogous to the Meissner effect in superconductors. Following on from Eq. (G3), where $\mathbf{E}=\mathbf{0}$ once equilibrium is reached, we have 


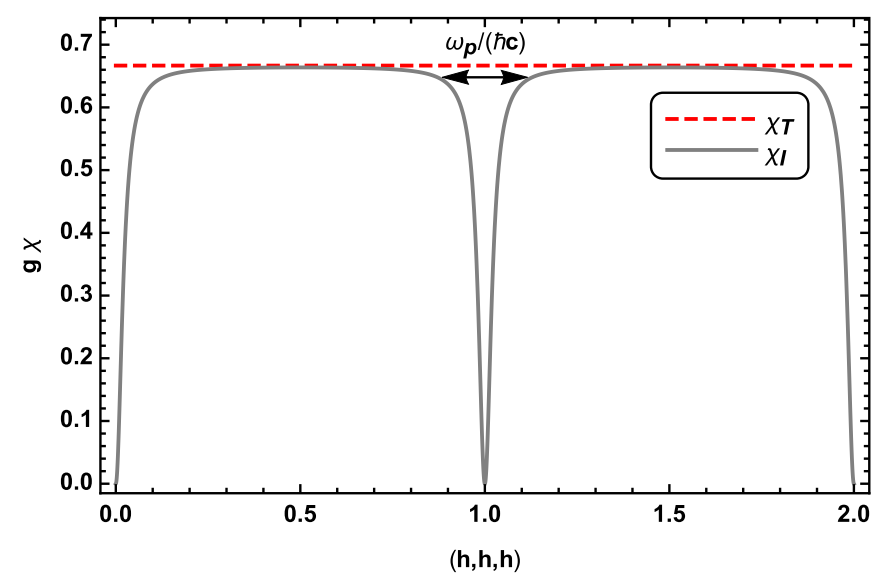

FIG. 7. The transverse isothermal $\left(\chi_{T}\right)$ and isolated $\left(\chi_{I}\right)$ susceptibilities along $\mathbf{k}=\frac{2 \pi}{a_{0}}(h, h, h)$ at $T=0.15 g$. The isolated susceptibility deviates from the isothermal one and decays to zero on length scales $\sim \omega_{p} /(\hbar c)$. This is because of persistent vison currents that are set up as the external magnetic field is switched on, whose magnetic fields oppose the applied field.

the following screening equation

$$
\nabla^{2} \mathbf{A}=\frac{\omega_{p}^{2}}{c^{2} \hbar^{2}} \mathbf{A},
$$

where $c \hbar / \omega_{p}$ is the characteristic screening length. Note that, in contrast to superconductors, this corresponds to the screening of the 'magnetic field' defined as $B_{i j} \equiv S_{i j}^{z}$, not the physical applied field, which is not eliminated.

Measuring the isolated susceptibility thus provides a possible experimental verification of the presence of dynamical visons in the system. In particular, if a uniform field $h$ along the global $x$ direction is applied, the perturbation takes the form

$$
\begin{aligned}
\delta \hat{H} & =-h \eta \sum_{\mathbf{r}, \mu} \hat{S}_{\mu}^{z}\left(\mathbf{r}+\mathbf{e}_{\mu} / 2\right)\left(\hat{\mathbf{e}}_{\mu} \cdot \hat{\mathbf{e}}_{x}\right) \\
& =\frac{2 h \eta \sqrt{N_{s}}}{3}\left(S_{\lambda=1}^{z}(\mathbf{k}=\mathbf{0})-S_{\lambda=2}^{z}(\mathbf{k}=\mathbf{0})\right),
\end{aligned}
$$

where $\eta$ is the individual spin's magnetic moment. The resulting magnetization per lattice site, along the global $x$ direction, is given by

$$
\begin{aligned}
M_{x} & \equiv \frac{\eta}{4 N_{s}} \sum_{\mathbf{r}, \mu}\left\langle\hat{S}_{\mu}^{z}\left(\mathbf{r}+\mathbf{e}_{\mu} / 2\right)\right\rangle\left(\hat{\mathbf{e}}_{\mu} \cdot \hat{\mathbf{e}}_{x}\right) \\
& = \begin{cases}\frac{2 \eta^{2} h \chi_{1}^{I}(\mathbf{0})}{9}=0 & \text { isolated, } \\
\frac{2 \eta^{2} h \chi_{1}^{T}(\mathbf{0})}{9}=\frac{\eta^{2} h}{18 g s^{4} z} & \text { isothermal. }\end{cases}
\end{aligned}
$$

Thus visons lead to the vanishing of the uniform isolated susceptibility. In fact, it is a straightforward generalization of the above to show that visons cause the uniform ac susceptibility of an isolated system to vanish as long as the frequency of the applied field is different from the plasma frequency.

\section{E. Heat capacity}

We have also investigated the effect of the mass gap on the photon contribution to the heat capacity. As in the case

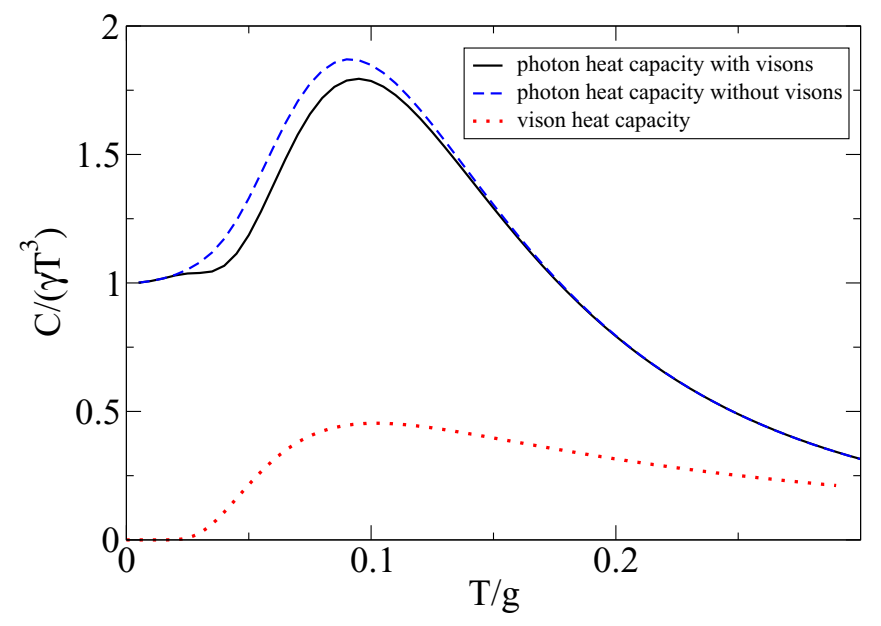

FIG. 8. The photonic contribution to the heat capacity per unit volume with and without visons. Note that because the visongenerated photon mass is suppressed exponentially at low temperatures, it has no effect on the $\gamma T^{3}$ behavior of the heat capacity in this region $\left[\gamma=\frac{4 \pi^{2}}{15(c \hbar)^{3}}\right]$. By equipartition theorem, the photon heat capacity tends to $8 a_{0}^{-3}$ at high temperatures regardless of the presence of the mass gap. Hence, the effects of the mass gap are most pronounced at intermediate temperatures.

of the equal-time structure factor, the dynamical mass gap has a small effect on the heat capacity. Because the visons are thermally activated, they are exponentially suppressed at small temperatures and do not affect the low-temperature $T^{3}$ behavior of the photonic heat capacity. At high temperatures, the mass gap does not affect the equipartition limit of the photon heat capacity, i.e., $k_{B}$ per mode. There is only a small difference between the photonic heat capacity with and without visons at intermediate temperatures. Figure 8 demonstrates this (see Appendix $\mathrm{E}$ for the derivation of the heat capacity). The contribution of the vison gas to the heat capacity is also included for comparison.

\section{F. Confinement of spinons}

We note that the dynamical mass gap generated by the vison plasma does not cause confinement of magnetic monopoles (spinons). Introducing a pair of magnetic monopoles into the system corresponds to choosing an appropriate static background field $A_{\lambda}^{0}(\mathbf{k})$, which only couples to the zero Matsubara frequency component of the dynamical gauge field $A_{\lambda}(\mathbf{k}, \omega=0)$, which is not gapped. Hence, the vison plasma does not alter the Coulomb interaction between static magnetic monopoles introduced into the system. We refer the reader to Appendix F for a detailed mechanism of how a static mass gap, e.g., one generated by the condensation of visons in the ground state, causes confinement of magnetic monopoles.

\section{CONCLUSION}

We have described some of the possible experimental signatures of gapped excitations of QSI, known as visons. Because visons are sources of emergent electric field, they are not easily accessible to experimental probes. Perhaps, magnetostriction or Dzyaloshinskii-Moriya effects can couple 
real electric fields to the emergent electric field of the visons [26,34-36], in which case visons can leave a signature in electric response measurements. In this paper, we have instead focused on how the dynamics of the vison's emergent electric field couple to the dynamics of the physical magnetic field, via electromagnetic induction. This coupling leaves signatures in neutron scattering experiments, which probe the magnetic field dynamics.

We have been able to capture the dynamics of the visons analytically and quantitatively for the first time by studying the ring-exchange Hamiltonian in the large- $s$ limit. We extended the perturbative analysis of our previous work [9] by including nonperturbative corrections that correspond to the tunneling of visons between lattice sites. These quantum corrections also go beyond the recent classical description of visons in Ref. [26] and endow them with a finite mass (exponentially small in $s$ ), which we have calculated.

In agreement with previous works, we have shown that visons give rise to a continuum of excitations above the ground state and renormalize the speed of light. Both were calculated in the large-s limit. What is perhaps more novel, is that in the large- $s$ description, as soon as the temperature becomes nonzero, the visons have a dramatic effect on the photon spectrum. The visons form a dilute 3D Coulomb gas, which, as is well known, is always in the plasma phase, i.e., the visons are deconfined. We have investigated how this plasma interacts with the long-wavelength degrees of freedom of the gauge field. We have found that in the Debye limit, where a quadratic description is viable, plasma oscillations introduce a dynamical mass gap in the photon spectrum, which follows an Arrhenius law at low temperatures. This mass gap should be observable in inelastic magnetic response measurements. In particular, it should show up in energyresolved neutron-scattering experiments and we have calculated the scattering intensity as would be seen in a typical experiment. We have compared our scattering intensity results with the recent quantum Monte Carlo (QMC) simulations of Ref. [20]. These are in rough agreement, although, because of the limited resolution of QMC, the linear photon dispersion cannot be resolved, let alone a potential small gap in the spectrum.

We also show that the photon mass gap generated by visons does not couple to the zero Matsubara frequency component of the gauge field, and hence does not result in confinement of static magnetic monopoles (spinons) introduced into the system. However, it leads to the vanishing of the uniform susceptibility of an isolated system through a mechanism analogous to the Meissner effect. In fact, visons will lead to the vanishing of the uniform ac susceptibility as the long as the system is isolated and the frequency of the applied field is different from the plasma frequency.

An interesting question to consider is the effect of the photon mass gap on dynamical spinons. We leave this to be fully addressed by future works, but note here that induced vison currents are expected to lead to the decay of coherent spinon excitations by Lenz's law. The induced currents will also cause the Coulombic magnetic field of a spinon, which is propagating nonrelativistically and at constant speed, to form a flux tube of radius $(c \hbar) / \omega_{p}$ on a timescale of $\hbar / \omega_{p}$. Visons will thus lead to confinement of propagating spinons and the destruction of the Coulombic quantum spin liquid. Our large- $s$ description predicts this to happen as soon as the temperature becomes nonzero due to the development of a finite, albeit exponentially small, density of visons. There is thus a smooth cross-over between the Coulombic quantum spin liquid at zero temperature and the paramagnetic phase at higher temperatures, i.e., the above spinon confinement length scale and timescale diverge exponentially as we approach zero temperature. This is in contrast to the gauge mean-field theory analysis of Ref. [23], which predicts a first order transition between these two phases, even in the perturbative $J_{z} \gg J_{ \pm}$ limit that we have considered in this work.

\section{ACKNOWLEDGMENTS}

This work was supported in part by UKRI Grant Nos. EP/K028960/1 and EP/P013449/1, and by the UKRI NetworkPlus on "Emergence and Physics far from Equilibrium." We gratefully acknowledge discussions with C. Castelnovo, A. Szabó, and G. Goldstein.

\section{APPENDIX A: THE LONGITUDINAL AND TRANSVERSE PARTS OF THE INSTANTON SOLUTION IN THE CONTINUUM LIMIT}

It is very insightful to consider the continuum limit of the instanton solution in Eq. (38). We first write down the time-dependent part of the instanton solution as a sum of longitudinal and transverse parts

$$
E_{\mu}^{\text {inst. }}(\mathbf{r}, \tau)-E_{\mu}^{\text {const. }}(\mathbf{r})=E_{\mu}^{\text {tran. }}(\mathbf{r}, \tau)+E_{\mu}^{\text {long. }}(\mathbf{r}, \tau),
$$

where by definition $\operatorname{div}_{\alpha} E^{\text {tran. }}(\tau)=0$ and $\operatorname{curl}_{i j} E^{\text {long. }}(\tau)=0$ everywhere. From the solution in Eq. (38), it follows that

$$
\begin{aligned}
E_{\mu}^{\text {tran. }}(\mathbf{r}, \tau)= & \frac{1}{N_{s}} \sum_{\lambda=1,2, \mathbf{k} \in \mathrm{BZ}} \int_{-\infty}^{\infty} d \omega \frac{-i \omega e^{-i \omega \tau+i \mathbf{k} \cdot \mathbf{r}}}{\frac{2 z \tilde{g}^{2}}{s^{2}} \xi_{\lambda}^{2}(\mathbf{k})+\frac{\omega^{2}}{2}} \\
& \times U_{\mu \lambda}^{\dagger}(\mathbf{k}) U_{\lambda \sigma}(\mathbf{k}) \\
\underset{|\mathbf{k}| a_{0} \ll 1}{\approx} \int \frac{a_{0}^{3} d^{3} \mathbf{k}}{4(2 \pi)^{3}} \int_{0}^{\infty} d \omega \frac{-i \omega e^{-i \omega \tau+i \mathbf{k} \cdot \mathbf{r}}}{\left(\frac{2 z \tilde{g}^{2} a_{0}^{2}}{s^{2}}|\mathbf{k}|^{2}+\frac{\omega^{2}}{2}\right)} & \\
& \times \frac{3}{4}\left[\hat{\mathbf{e}}_{\sigma} \cdot \hat{\mathbf{e}}_{\mu}-\left(\hat{\mathbf{k}} \cdot \hat{\mathbf{e}}_{\sigma}\right)\left(\hat{\mathbf{k}} \cdot \hat{\mathbf{e}}_{\mu}\right)\right],
\end{aligned}
$$

where we have used the identity

$$
\begin{aligned}
\sum_{\lambda=1,2} U_{\mu \lambda}^{\dagger}(\mathbf{k}) U_{\lambda \sigma}(\mathbf{k}) & =\frac{1}{\xi_{1}^{2}(\mathbf{k})} \sum_{\lambda} U_{\mu \lambda}^{\dagger}(\mathbf{k}) \xi_{\lambda}^{2}(\mathbf{k}) U_{\lambda \sigma}(\mathbf{k}) \\
& =\frac{1}{\xi_{1}^{2}(\mathbf{k})} \sum_{\nu} Z_{\mu \nu}(\mathbf{k}) Z_{\nu \sigma}(\mathbf{k}) \\
& \stackrel{\mathbf{k} a_{0} \ll 1}{\approx} \frac{-4}{|\mathbf{k}|^{2} a_{0}^{2}} \sum_{\nu}\left(\mathbf{k} \cdot \Delta_{\mu \nu}\right)\left(\mathbf{k} \cdot \Delta_{\nu \sigma}\right) \\
& =\frac{3}{4}\left(\left(\hat{\mathbf{e}}_{\mu} \cdot \hat{\mathbf{e}}_{\sigma}\right)-\left(\hat{\mathbf{k}} \cdot \hat{\mathbf{e}}_{\mu}\right)\left(\hat{\mathbf{k}} \cdot \hat{\mathbf{e}}_{\sigma}\right)\right) .
\end{aligned}
$$

The transverse part of the instanton solution is transient in time with a power-law tail $\propto 1 /|\tau|^{3}$ at long times. 
The longitudinal part of the electric field can also be extracted from the solution in Eq. (38)

$$
\begin{aligned}
& E_{\mu}^{\text {long. }}(\mathbf{r}, \tau)=-\frac{\pi}{N_{s}} \operatorname{sgn}(\tau) \sum_{\lambda=3,4, \mathbf{k} \in \mathrm{BZ}} e^{i \mathbf{k} \cdot \mathbf{r}} U_{\mu \lambda}^{\dagger}(\mathbf{k}) U_{\lambda \sigma}(\mathbf{k}) \\
& \stackrel{|\mathbf{k}| a_{0} \ll 1}{\approx} \frac{3 \pi}{4} \operatorname{sgn}(\tau) \int \frac{a_{0}^{3} d^{3} \mathbf{k}}{4(2 \pi)^{3}} e^{i \mathbf{k} \cdot \mathbf{r}} \\
& \\
& \times\left(\frac{1}{3}+\left(\hat{\mathbf{k}} \cdot \hat{\mathbf{e}}_{\sigma}\right)\left(\hat{\mathbf{k}} \cdot \hat{\mathbf{e}}_{\mu}\right)\right) \\
&= \frac{3 \pi a_{0}^{3}}{16} \operatorname{sgn}(\tau) \delta(\mathbf{r})+A \mathbf{E}(\mathbf{r}, \tau) \cdot \hat{\mathbf{e}}_{\mu}
\end{aligned}
$$

where the factor of $A=\frac{\sqrt{3}}{4} a_{0}^{2}$ links the lattice electric flux $E_{\mu}(\mathbf{r}, \tau)$ with the continuum flux density $\mathbf{E}(\mathbf{r}, \tau)$ and follows from approximating a sum over a large closed surface $\mathrm{S}$ by a surface integral $\sum_{(\mathbf{r}, \mu) \in \mathrm{S}} \rightarrow \oint_{\mathrm{S}} \frac{d S}{A} . \mathbf{E}(\mathbf{r}, \tau)$ is the continuum electric field due to a dipole at $\mathbf{r}=\mathbf{0}$ with a dipole moment of $\pi \operatorname{sgn}(\tau) \mathbf{e}_{\sigma}$

$$
\begin{aligned}
\mathbf{E}(\mathbf{r}, \tau) & =-\pi i \operatorname{sgn}(\tau) \nabla\left[\int \frac{d^{3} \mathbf{k}}{(2 \pi)^{3}} \frac{\mathbf{k} \cdot \mathbf{e}_{\sigma}}{|\mathbf{k}|^{2}} e^{i \mathbf{k} \cdot \mathbf{r}}\right] \\
& =\pi \operatorname{sgn}(\tau) \nabla\left[\mathbf{e}_{\sigma} \cdot \nabla\left(\frac{1}{|\mathbf{r}|}\right)\right] .
\end{aligned}
$$

We have also used the identity

$$
\begin{aligned}
\sum_{\lambda=3,4} U_{\mu \lambda}^{\dagger}(\mathbf{k}) U_{\lambda \sigma}(\mathbf{k}) & =\delta_{\mu \sigma}-\sum_{\lambda=1,2} U_{\mu \lambda}^{\dagger}(\mathbf{k}) U_{\lambda \sigma}(\mathbf{k}) \\
\stackrel{|\mathbf{k}| a_{0}}{\approx} & \frac{3}{4}\left(\frac{1}{3}+\left(\hat{\mathbf{k}} \cdot \hat{\mathbf{e}}_{\sigma}\right)\left(\hat{\mathbf{k}} \cdot \hat{\mathbf{e}}_{\mu}\right)\right) .
\end{aligned}
$$

The longitudinal part of the electric field is uniquely determined by the charge distribution via Laplace's equation in Eq. (30). Because $E_{\mu}^{\text {long. }}(\mathbf{r}, \tau)$ has a lattice divergence of $-\pi \operatorname{sgn}(\tau)$ at $-\mathbf{e}_{\mu} / 2$ and $\pi \operatorname{sgn}(\tau)$ at $\mathbf{e}_{\mu} / 2$, it necessarily corresponds to the electric field due to a pair of charges $\pi$ and $-\pi$ at positions $-\mathbf{e}_{\mu} / 2$ and $\mathbf{e}_{\mu} / 2$ respectively for $\tau<0$ that switch positions for $\tau>0$. We therefore obtain a dipole field as above in the continuum limit. It also follows that, to describe the hopping of a vison of charge $2 \pi$ at $\tau=0$, the constant background electric field $E_{\mu}^{\text {const. }}(\mathbf{r}, \tau)$ has to be the longitudinal field due to a pair of charges $\pi$ at positions $\pm \mathbf{e}_{\mu} / 2$.

\section{APPENDIX B: THE SMOOTH INSTANTON SOLUTION}

We begin with the instanton solution in Eq. (38) [in the following, the instanton solution is given relative to the constant and divergenceful background field $\left.E_{\lambda}^{\text {const. }}(\mathbf{k})\right]$

$E_{\lambda}^{\text {inst. }}(\mathbf{k}, \tau)=\frac{d}{d \tau}\left[\frac{1}{\sqrt{N_{s}} \beta} \sum_{\omega} \frac{\pi U_{\lambda \sigma}(\mathbf{k})}{\frac{2 z \tilde{g}^{2}}{s^{2}} \xi_{\lambda}^{2}(\mathbf{k})+\frac{\omega^{2}}{2}} e^{-i \omega \tau}\right]$.
Taking the low temperature limit $\beta \tilde{g} \sqrt{z} / s \rightarrow \infty$, we obtain

$$
\begin{aligned}
E_{\lambda}^{\text {inst. }}(\mathbf{k}, \tau) & =\frac{d}{d \tau} \int_{-\infty}^{\infty} \frac{d \omega}{2 \pi \sqrt{N_{s}}} \frac{\pi U_{\lambda \sigma}(\mathbf{k})}{\frac{2 z \tilde{g}^{2}}{s^{2}} \xi_{\lambda}^{2}(\mathbf{k})+\frac{\omega^{2}}{2}} e^{-i \omega \tau} \\
& =\frac{s \pi U_{\lambda \sigma}(\mathbf{k})}{2 \tilde{g} \sqrt{N_{s} z} \xi_{\lambda}(\mathbf{k})} \frac{d}{d \tau}\left[e^{-|\tau| \frac{2 \tilde{g} \sqrt{2}\left|\xi_{\lambda}(\mathbf{k})\right|}{s}}\right] \\
& =-\frac{\pi \operatorname{sgn}(\tau) U_{\lambda \sigma}(\mathbf{k})}{\sqrt{N_{s}}} e^{-|\tau| \frac{2 \tilde{g} \sqrt{z}\left|\xi_{\lambda}(\mathbf{k})\right|}{s}} .
\end{aligned}
$$

The smooth instanton solution in Eq. (42) is given by

$E_{\lambda}^{\mathrm{sm} .}(\mathbf{k}, \tau)=\frac{\pi U_{\lambda \sigma}(\mathbf{k})}{\sqrt{N_{s}}}\left[-\operatorname{sgn}(\tau) e^{-|\tau| \frac{2 \tilde{g} \sqrt{2}\left|\xi_{\lambda}(\mathbf{k})\right|}{s}}+\operatorname{sgn}(\tau)+1\right]$,

and we can see that the jump in the field has been removed. Straightforward differentiation gives

$$
\ddot{E}_{\lambda}^{\mathrm{sm} .}(\mathbf{k}, \tau)=-\frac{\pi \operatorname{sgn}(\tau) U_{\lambda \sigma}(\mathbf{k})}{\sqrt{N_{s}}} \frac{4 z \tilde{g}^{2} \xi_{\lambda}^{2}(\mathbf{k})}{s^{2}} e^{-|\tau| \frac{2 \tilde{g} \sqrt{z}\left|\xi_{\lambda}(\mathbf{k})\right|}{s}} .
$$

The smooth instanton solution is the stationary solution of the action in Eq. (41). Minimising that action with respect to variations in the dynamical, transverse part of the field $E_{\lambda=1,2}(-\mathbf{k}, \tau)$ we obtain the following saddle point equations

$$
\frac{-2 s^{2}}{4 z \tilde{g} \xi_{\lambda}^{2}(\mathbf{k})} \ddot{E}_{\lambda}(\mathbf{k}, \tau)+\tilde{g} \sum_{\mathbf{r}, \mu} V^{\prime}\left[E_{\mu}(\mathbf{r}, \tau)\right] \frac{\delta E_{\mu}(\mathbf{r}, \tau)}{\delta E_{\lambda}(-\mathbf{k}, \tau)}=0,
$$

for all $\mathbf{k}$ and $\lambda=1$ and 2 , where

$$
\frac{\delta E_{\mu}(\mathbf{r}, \tau)}{\delta E_{\lambda}(-\mathbf{k}, \tau)}=\frac{1}{\sqrt{N_{s}}} U_{\mu \lambda}^{\dagger}(-\mathbf{k}) e^{-i \mathbf{k} \cdot \mathbf{r}}
$$

and

$$
V^{\prime}\left[E_{\mu}(\mathbf{r}, \tau)\right]=2 E_{\mu}(\mathbf{r}, \tau)-2 \pi \delta_{\mu \sigma} \delta_{\mathbf{r}, \mathbf{0}}[\operatorname{sgn}(\tau)+1] .
$$

The first derivative of the continued parabolic potential $V^{\prime}\left[E_{\mu}(\mathbf{r}, \tau)\right]$ is given by $2 E_{\mu}(\mathbf{r}, \tau)$, when $\left|E_{\mu}(\mathbf{r}, \tau)\right| \leqslant \pi$. This is true in the case of the above smooth instanton solution $E_{\mu}^{\mathrm{sm} .}(\mathbf{r}, \tau)$, everywhere except for the single electric field $E_{\mu=\sigma}(\mathbf{r}=\mathbf{0}, \tau)$, which lies between $\pi$ and $3 \pi$ for positive imaginary times. In this case $V^{\prime}\left[E_{\mu}(\mathbf{r}, \tau)\right]=2 E_{\mu}(\mathbf{r}, \tau)-4 \pi$, thus justifying the above expression. It follows that the smooth instanton solution $E_{\mu}^{\mathrm{sm} .}(\mathbf{r}, \tau)$ satisfies the saddle point equation in Eq. (B5)

$$
\begin{aligned}
0= & -2 \ddot{E}_{\lambda}^{\mathrm{sm}} \cdot(\mathbf{k}, \tau) \frac{s^{2}}{4 z \tilde{g} \xi_{\lambda}^{2}(\mathbf{k})}+2 \tilde{g} E_{\lambda}^{\mathrm{sm} .}(\mathbf{k}, \tau) \\
& -\frac{2 \pi \tilde{g}}{\sqrt{N_{s}}} U_{\lambda \sigma}(\mathbf{k})[\operatorname{sgn}(\tau)+1] .
\end{aligned}
$$

We now turn to proving that $\dot{E}_{\lambda}^{\text {sm. }}(\mathbf{k}, \tau)$ is the zero-energy mode of the action in Eq. (43) describing fluctuations around 
the stationary smooth instanton solution

$$
\begin{aligned}
\delta \mathcal{S}_{\operatorname{tran}}\left[\delta E_{\lambda}(\mathbf{k}, \tau)\right]= & \int_{-\beta / 2}^{\beta / 2} d \tau \sum_{\mathbf{k} \in \mathrm{BZ}, \lambda=1,2} \frac{s^{2}\left|\delta \dot{E}_{\lambda}(\mathbf{k}, \tau)\right|^{2}}{4 z \tilde{g} \xi_{\lambda}^{2}(\mathbf{k})} \\
& +\int_{-\beta / 2}^{\beta / 2} d \tau \frac{\tilde{g}}{2} \sum_{\alpha \beta} V^{\prime \prime}\left(E_{\alpha \beta}^{\mathrm{sm} .}(\tau)\right) \delta E_{\alpha \beta}^{2}(\tau) .
\end{aligned}
$$

The zero-mode of the above action satisfies the following differential equation for $\lambda=1$ and 2 :

$$
\begin{aligned}
0= & -\frac{s^{2}}{4 z \tilde{g} \xi_{\lambda}^{2}(\mathbf{k})} \delta \ddot{E}_{\lambda}(\mathbf{k}, \tau) \\
& +\frac{\delta E_{\mu}(\mathbf{r}, \tau)}{\delta E_{\lambda}(-\mathbf{k}, \tau)} \frac{\tilde{g}}{2} \sum_{\alpha \beta} V^{\prime \prime}\left[E_{\alpha \beta}^{\mathrm{sm}} \cdot(\tau)\right] \delta E_{\alpha \beta}(\tau) .
\end{aligned}
$$

We see that $\delta E_{\alpha \beta}(\tau)=\dot{E}_{\alpha \beta}^{\mathrm{sm}}$. $(\tau)$ is a solution of the equation, because

$$
-\frac{s^{2}}{4 z \tilde{g} \xi_{\lambda}^{2}(\mathbf{k})} \dddot{E}_{\lambda}^{\mathrm{sm} .}(\mathbf{k})+\frac{\delta E_{\mu}(\mathbf{r}, \tau)}{\delta E_{\lambda}(-\mathbf{k}, \tau)} \frac{\tilde{g}}{2} \sum_{\alpha \beta} V^{\prime \prime}\left[E_{\alpha \beta}^{\mathrm{sm} .}(\tau)\right] \dot{E}_{\alpha \beta}^{\mathrm{sm} .}(\tau)
$$

is proportional to the time derivative of the left-hand side of Eq. (B5).

\section{APPENDIX C: INSTANTON MEASURE}

The fluctuation action in Eq. (43) can be written as follows:

$$
\begin{aligned}
\delta S= & \sum_{\omega} \sum_{\mathbf{k}, \lambda=1,2}\left(\tilde{g}+\frac{s^{2} \omega^{2}}{4 z \tilde{g} \xi_{\lambda}^{2}(\mathbf{k})}\right)\left|\delta E_{\lambda}(\mathbf{k}, \omega)\right|^{2} \\
& -\frac{2 \pi \tilde{g}}{\dot{E}_{\sigma}^{\mathrm{sm}} \cdot(\mathbf{0}, 0)} \delta E_{\sigma}^{2}(\mathbf{0}, 0),
\end{aligned}
$$

where

$$
\begin{aligned}
\dot{E}_{\sigma}^{\mathrm{sm} .}(0, \mathbf{0}) & =\frac{2 \sqrt{z} \pi \tilde{g}}{s N_{s}} \sum_{\mathbf{k}, \lambda} U_{\sigma \lambda}^{\dagger}(\mathbf{k})\left|\xi_{\lambda}(\mathbf{k})\right| U_{\lambda \sigma}(\mathbf{k}) \\
& =\frac{2 \sqrt{z} \pi \tilde{g}}{s N_{s}} \sum_{\mathbf{k} \in \text { B.Z., } \mu} \frac{Z_{\sigma \mu}(\mathbf{k}) Z_{\mu \sigma}(\mathbf{k})}{\left|\xi_{1}(\mathbf{k})\right|} \\
& \equiv \frac{C_{3}^{2} \tilde{g}}{s}
\end{aligned}
$$

and $C_{3}=5.13$. It is convenient to express the fluctuation action in matrix form

$$
\delta S=\mathbf{E}^{\dagger} \tilde{\mathbf{K}} \mathbf{E}=\mathbf{E}^{\dagger}\left(\mathbf{K}-\lambda \mathbf{v} \mathbf{v}^{\dagger}\right) \mathbf{E},
$$

where

$$
\begin{aligned}
{[\mathbf{E}]_{\lambda \omega \mathbf{k}} } & =\delta E_{\lambda}(\omega, \mathbf{k}), \\
{[\mathbf{v}]_{\lambda \omega \mathbf{k}} } & =\frac{1}{\sqrt{N_{s} \beta}} U_{\lambda \sigma}(\mathbf{k}), \\
{[\mathbf{K}]_{\lambda \omega \mathbf{k}, \lambda^{\prime} \omega^{\prime} \mathbf{k}^{\prime}} } & =\delta_{\lambda \omega \mathbf{k}, \lambda^{\prime} \omega^{\prime} \mathbf{k}^{\prime}}\left(\tilde{g}+\frac{s^{2} \omega^{2}}{4 z \tilde{g} \xi_{\lambda}^{2}(\mathbf{k})}\right), \\
\lambda & =\frac{2 \pi s}{C_{3}^{2}}=\frac{1}{\mathbf{v}^{\dagger} \mathbf{K}^{-1} \mathbf{v}},
\end{aligned}
$$

where the final identity follows from Eq. (C2).
The instanton measure can be obtained by working out the contribution of the one-instanton sector to the partition function relative to the zero-instanton sector

$$
\begin{gathered}
\frac{\int \prod_{i} d \tilde{\xi}_{i} \sqrt{\left\langle\tilde{\Psi}_{i} \mid \tilde{\Psi}_{i}\right\rangle} e^{-\frac{1}{2} \sum_{i \neq 0} \tilde{\lambda}_{i} \tilde{\xi}_{i}^{2}\left\langle\tilde{\Psi}_{i} \mid \tilde{\Psi}_{i}\right\rangle}}{\int \prod_{i} d \xi_{i} \sqrt{\left\langle\Psi_{i} \mid \Psi_{i}\right\rangle} e^{-\frac{1}{2} \sum_{i} \lambda_{i} \xi_{i}^{2}\left\langle\Psi_{i} \mid \Psi_{i}\right\rangle}} \\
=\int d \tilde{\xi}_{0} \sqrt{\frac{\left\langle\tilde{\Psi}_{0} \mid \tilde{\Psi}_{0}\right\rangle}{2 \pi}} \sqrt{\frac{\operatorname{det} \mathbf{K}}{\operatorname{det}^{\prime} \tilde{\mathbf{K}}}}
\end{gathered}
$$

where we have written down $\delta E(\mathbf{r}, \tau)=\sum_{i} \xi_{i} \Psi_{i}(\mathbf{r}, \tau)=$ $\sum_{i} \tilde{\xi}_{i} \tilde{\Psi}_{i}(\mathbf{r}, \tau)$ in terms of the real eigenvectors of $\mathbf{K}$ and $\tilde{\mathbf{K}}$ respectively in the $(\mathbf{r}, \tau)$ basis and the zero eigenvalue is excluded from the determinant of $\tilde{\mathbf{K}}$. From

$$
\tilde{\mathbf{K}}=\mathbf{K}\left(\mathbf{1}-\lambda \mathbf{K}^{-1} \mathbf{v} \mathbf{v}^{\dagger}\right),
$$

it follows that

$$
\frac{\operatorname{det}(\tilde{\mathbf{K}})}{\operatorname{det}(\mathbf{K})}=\operatorname{det}\left(\mathbf{1}-\lambda \mathbf{K}^{-1} \mathbf{v} \mathbf{v}^{\dagger}\right) \text {. }
$$

The matrix $\left(\mathbf{1}+\lambda \mathbf{K}^{-1} \mathbf{v} \mathbf{v}^{\dagger}\right)$ has $N-1$ eigenvectors perpendicular to $\mathbf{v}$ with eigenvalue 1 and an eigenvector $\mathbf{K}^{-1} \mathbf{v}$ with eigenvalue equal to $\left(1-\lambda \mathbf{v}^{\dagger} \mathbf{K}^{-1} \mathbf{v}\right)$. Therefore

$$
\frac{\operatorname{det}(\tilde{\mathbf{K}})}{\operatorname{det}(\mathbf{K})}=\left(1-\lambda \mathbf{v}^{\dagger} \mathbf{K}^{-1} \mathbf{v}\right) \text {. }
$$

When $\lambda$ takes on its physical value given by Eq. (C4) and equal to $\left(\mathbf{v}^{\dagger} \mathbf{K}^{-1} \mathbf{v}\right)^{-1}$, det $\tilde{\mathbf{K}}$ and the above ratio vanish. To avoid this, we will perturb $\lambda$ from its physical value as follows

$$
\lambda=\frac{1}{\mathbf{v}^{\dagger} \mathbf{K}^{-1} \mathbf{v}}-\delta \lambda \text {. }
$$

The matrix $\tilde{\mathbf{K}}$ changes as a result by $\delta \tilde{\mathbf{K}}=\delta \lambda \mathbf{v} \mathbf{v}^{\dagger}$, and standard perturbation theory gives the shift of the zero eigenvalue of $\tilde{\mathbf{K}}$

$$
\delta \tilde{\lambda}_{0}=\frac{\left\langle\mathbf{K}^{-1} \mathbf{v}|\delta \tilde{\mathbf{K}}| \mathbf{K}^{-1} \mathbf{v}\right\rangle}{\left\langle\mathbf{K}^{-1} \mathbf{v} \mid \mathbf{K}^{-1} \mathbf{v}\right\rangle}=\frac{\left(\mathbf{v}^{\dagger} \mathbf{K}^{-1} \mathbf{v}\right)^{2} \delta \lambda}{\mathbf{v}^{\dagger} \mathbf{K}^{-2} \mathbf{v}} .
$$

With the perturbed $\lambda$, we can now evaluate

$$
\frac{\operatorname{det}^{\prime}(\tilde{\mathbf{K}})}{\operatorname{det}(\mathbf{K})}=\frac{\operatorname{det}(\tilde{\mathbf{K}})}{\delta \tilde{\lambda}_{0} \operatorname{det}(\mathbf{K})}=\frac{\mathbf{v}^{\dagger} \mathbf{K}^{-1} \mathbf{v} \delta \lambda}{\delta \tilde{\lambda}_{0}}=\frac{\mathbf{v}^{\dagger} \mathbf{K}^{-2} \mathbf{v}}{\mathbf{v}^{\dagger} \mathbf{K}^{-1} \mathbf{v}}
$$

and then take the limit $\delta \lambda \rightarrow 0$, where

$$
\begin{aligned}
\mathbf{v}^{\dagger} \mathbf{K}^{-2} \mathbf{v} & =\frac{1}{N_{s} \beta} \sum_{\omega} \sum_{\mathbf{k}, \lambda} U_{\sigma \lambda}^{\dagger}(\mathbf{k})\left(\frac{1}{\tilde{g}+\frac{\omega^{2} s^{2}}{4 z \tilde{g} \xi_{\lambda}^{2}(\mathbf{k})}}\right)^{2} U_{\lambda \sigma}(\mathbf{k}) \\
& =\frac{C_{3}^{2}}{4 \pi \tilde{g} s}
\end{aligned}
$$

and we have summed over $\omega$ in the $\beta \tilde{g} \sqrt{z} / s \rightarrow \infty$ limit and used the identity quoted in Eq. (C2).

We also calculate the norm of the zero mode

$$
\begin{aligned}
\left\langle\tilde{\Psi}_{0} \mid \tilde{\Psi}_{0}\right\rangle & =\sum_{\mathbf{k}, \lambda} \int_{-\infty}^{\infty}\left|\dot{E}_{\lambda}^{\mathrm{sm}} \cdot(\tau, \mathbf{k})\right|^{2} d \tau \\
& =\frac{2 \sqrt{z} \tilde{g} \pi^{2}}{N_{s} s} \sum_{\mathbf{k}, \lambda} \frac{U_{\sigma \lambda}^{\dagger} \xi_{\lambda}^{2}(\mathbf{k}) U_{\lambda \sigma}(\mathbf{k})}{\left|\xi_{1}(\mathbf{k})\right|} \\
& =\frac{C_{3}^{2} \pi \tilde{g}}{s},
\end{aligned}
$$


where we have again used the identity quoted in Eq. (C2). Inserting everything into Eq. (C5), the instanton measure becomes

$$
\int \frac{C_{3} \tilde{g} d \tilde{\xi}_{0}}{\sqrt{s}}
$$

where $\xi_{0}$ specifies the position of the instanton in imaginary time.

\section{APPENDIX D: CONTRIBUTION OF VISON PAIRS}

We will look at the contribution of vison pairs to the $S^{z}$ correlator in the ground state.

\section{Renormalization of the speed of light}

In the large-s limit, virtual vison pairs will be far away from each other because each vison pair loop is accompanied by at least two instantons and will enter the partition function with a weight at least as small as $\sim e^{-2 \mu_{l}}=e^{-2 C_{2} s}$. We can therefore neglect the hardcore interactions between virtual vison pairs. Following the action in Eq. (49), the partition function of a dilute gas of such loops can be written as

$$
\begin{aligned}
Z= & \int d A_{\alpha \beta}(\tau) e^{-S_{0}} \sum_{n} \frac{1}{n !} \\
& \times\left(\sum_{\alpha \beta} \iint \frac{w^{2} d T d \tau}{e^{\mu_{V} T} e^{2 \mu_{I}}} e^{2 \pi i A_{\alpha \beta}\left(\tau-\frac{T}{2}\right)-2 \pi i A_{\alpha \beta}\left(\tau+\frac{T}{2}\right)}+\text { c.c. }\right)^{n},
\end{aligned}
$$

where $n$ is the number of loops considered, $T$ is the length of the loop in imaginary time, $\tau$ the position of its center and $w=\frac{C_{3} \tilde{g}}{\sqrt{s}}$ the instanton measure. As we are focusing on the effect on the $A_{\alpha \beta}(\tau)$ gauge field only, we have omitted the scalar field $\varphi_{\gamma}(\tau)$ from the above partition function. The contribution of vison pairs to the gauge field action, at quadratic order, can thus be written as

$$
\begin{aligned}
\delta S_{\text {pairs }}= & \frac{4 \pi^{2} w^{2}}{e^{2 \mu_{I}}} \sum_{\alpha \beta} \iint d T d \tau e^{-\mu_{V} T} \\
& \times\left(A_{\alpha \beta}\left(\tau+\frac{T}{2}\right)-A_{\alpha \beta}\left(\tau-\frac{T}{2}\right)\right)^{2} \\
= & \frac{16 \pi^{2} w^{2}}{e^{2 \mu_{I}}} \sum_{\omega, \mathbf{k}, \lambda=1,2} \frac{\omega^{2}\left|A_{\lambda}(\mathbf{k}, \omega)\right|^{2}}{4 \mu_{V}^{2}+\omega^{2}}
\end{aligned}
$$

$$
=\frac{4 \pi^{2} w^{2}}{\mu_{V}^{3} e^{2 \mu_{I}}} \sum_{\omega, \mathbf{k}, \lambda=1,2} \omega^{2}\left|A_{\lambda}(\mathbf{k}, \omega)\right|^{2} \quad \text { for } \omega \tilde{g} \ll 1 .
$$

We can see that virtual vison pairs lead to a small renormalization of the $\omega^{2}$ coefficient in the gauge field action $S_{0}$, and hence a small renormalization of the speed of light. We can also see that vison pair loops introduce a pole in $\chi_{\lambda}(\mathbf{k}, \omega)$ at $\omega=2 \mu_{V}$ corresponding to the energy of creation of a single static vison-antivison pair. To obtain a continuum of excitations above this threshold, we need to consider vison pair loops accompanied by more than two instantons, i.e., the visons execute hops before annihilating. It will be more convenient to do this using the $\mathrm{O}(2)$ rotor description of visons given in Eq. (51).

\section{Continuum of excitations above the ground state}

There will be a continuum of excitations at energies above twice the vison energy $2 \mu_{V}$. In the large-s limit, the ground state of the $\mathrm{O}(2)$ action in Eq. (51) is characterized by $q_{\gamma}(\tau)=0$ everywhere with small virtual vison fluctuations as a perturbation. In the same limit, the multi-particle excited states are described by the action in Eq. (53), as long as the excitations are dilute. The excited states consist of bosons of two flavors (corresponding to visons and antivisons). For small momenta and gauge fields, these excitations are described by the action in Eq. (54). The two-particle excited states are then connected to the ground state via the following perturbation, which creates or annihilates vison pairs

$$
\begin{aligned}
\delta S_{ \pm}= & 2 \pi v \int d^{3} \mathbf{r} \int d \tau\left(i \Psi^{-} \mathbf{A} \cdot \nabla \Psi^{+}+i \bar{\Psi}^{+} \mathbf{A} \cdot \nabla \bar{\Psi}^{-}+\text {H.c. }\right), \\
= & \frac{-2 \pi v}{\sqrt{\beta}} \sum_{\omega_{1}, \omega_{2}} \int \frac{d^{3} \mathbf{k}_{1} d^{3} \mathbf{k}_{2}}{(2 \pi)^{6}}\left(\Psi^{+}\left(\mathbf{k}_{1}, \omega_{1}\right) \Psi^{-}\left(\mathbf{k}_{2}, \omega_{2}\right)\right. \\
& \left.\times\left(\mathbf{k}_{1}-\mathbf{k}_{2}\right) \cdot \mathbf{A}\left(-\mathbf{k}_{1}-\mathbf{k}_{2},-\omega_{1}-\omega_{2}\right)+\text { c.c. }\right) \quad(\text { D3 })
\end{aligned}
$$

where $v=\frac{4 \hbar^{2}}{m^{*} a_{0}^{3}}$. The above perturbation gives a correction to the gauge field correlator $\left\langle A_{\lambda}(\mathbf{k}, \omega) A_{\lambda^{\prime}}\left(\mathbf{k}^{\prime}, \omega^{\prime}\right)\right\rangle$. The zeroth order correlator can be found using the continuum limit of the action $S_{0}$ given in Appendix G

$$
\left\langle A_{\lambda}(\mathbf{k}, \omega) A_{\lambda^{\prime}}\left(\mathbf{k}^{\prime}, \omega^{\prime}\right)\right\rangle_{0}=\frac{2 \tilde{g}(2 \pi)^{3} \delta^{3}\left(\mathbf{k}+\mathbf{k}^{\prime}\right) \delta_{\omega,-\omega^{\prime}} \delta_{\lambda \lambda^{\prime}}}{\omega^{2}+c^{2} \hbar^{2} \mathbf{k}^{2}} .
$$

Standard perturbation theory then gives the following lowest order correction to the gauge field correlator

$$
\begin{aligned}
\delta\left\langle A_{\lambda}(\mathbf{k}, \omega) A_{\lambda^{\prime}}\left(\mathbf{k}^{\prime}, \omega^{\prime}\right)\right\rangle= & \frac{\left\langle\left.\left(\delta S_{ \pm}\right)^{2} A_{\lambda}(\mathbf{k}, \omega) A_{\lambda^{\prime}}\left(\mathbf{k}^{\prime}, \omega^{\prime}\right)\right|_{0} ^{\mathrm{c}}\right.}{2} \\
= & \frac{4 \pi v^{2}}{\beta} \sum_{\omega_{1}, \omega_{2}, \Omega_{1}, \Omega_{2}} \int \frac{d^{3} \mathbf{k}_{1} d^{3} \mathbf{k}_{2} d^{3} \mathbf{q}_{1} d^{3} \mathbf{q}_{2}}{(2 \pi)^{12}}\left\langle\bar{\Psi}^{+}\left(\mathbf{q}_{1}, \Omega_{1}\right) \Psi^{+}\left(\mathbf{k}_{1}, \omega_{1}\right)\right\rangle_{0}\left\langle\bar{\Psi}^{-}\left(\mathbf{q}_{2}, \Omega_{2}\right) \Psi^{-}\left(\mathbf{k}_{2}, \omega_{2}\right)\right\rangle_{0} \\
& \times\left\langle A_{\lambda}(\mathbf{k}, \omega) A_{\lambda^{\prime}}\left(\mathbf{k}^{\prime}, \omega^{\prime}\right)\left(\mathbf{k}_{1}-\mathbf{k}_{2}\right) \cdot \mathbf{A}\left(-\mathbf{k}_{1}-\mathbf{k}_{2},-\omega_{1}-\omega_{2}\right)\left(\mathbf{q}_{1}-\mathbf{q}_{2}\right) \cdot \mathbf{A}\left(\mathbf{q}_{1}+\mathbf{q}_{2}, \Omega_{1}+\Omega_{2}\right)\right\rangle_{0}^{\mathrm{c}}
\end{aligned}
$$




$$
\begin{aligned}
= & \frac{4 \pi v^{2}}{\beta} \sum_{\omega_{1}, \omega_{2}} \int \frac{d^{3} \mathbf{k}_{1} d^{3} \mathbf{k}_{2}}{(2 \pi)^{6}} \frac{a_{0}^{3} / 8}{-i \omega_{1}+\epsilon\left(\mathbf{k}_{1}\right)} \frac{a_{0}^{3} / 8}{-i \omega_{2}+\epsilon\left(\mathbf{k}_{2}\right)}\left\langle A_{\lambda}(\mathbf{k}, \omega) A_{\lambda^{\prime}}\left(\mathbf{k}^{\prime}, \omega^{\prime}\right)\left(\mathbf{k}_{1}-\mathbf{k}_{2}\right)\right. \\
& \left.\cdot \mathbf{A}\left(-\mathbf{k}_{1}-\mathbf{k}_{2},-\omega_{1}-\omega_{2}\right)\left(\mathbf{k}_{1}-\mathbf{k}_{2}\right) \cdot \mathbf{A}\left(\mathbf{k}_{1}+\mathbf{k}_{2}, \omega_{1}+\omega_{2}\right)\right\rangle_{0}^{\mathrm{c}} \\
= & \frac{4 \pi v^{2}}{\beta} \sum_{\omega_{1}, \omega_{2}} \int \frac{d^{3} \mathbf{k}_{1} d^{3} \mathbf{k}_{2}}{(2 \pi)^{6}} \frac{a_{0}^{3} / 8}{-i \omega_{1}+\epsilon\left(\mathbf{k}_{1}\right)} \frac{a_{0}^{3} / 8}{i \omega_{1}-i \omega_{2}+\epsilon\left(\mathbf{k}_{2}-\mathbf{k}_{1}\right)} \\
& \times\left\langle A_{\lambda}(\mathbf{k}, \omega) A_{\lambda^{\prime}}\left(\mathbf{k}^{\prime}, \omega^{\prime}\right)\left(2 \mathbf{k}_{1}-\mathbf{k}_{2}\right) \cdot \mathbf{A}\left(-\mathbf{k}_{2},-\omega_{2}\right)\left(2 \mathbf{k}_{1}-\mathbf{k}_{2}\right) \cdot \mathbf{A}\left(\mathbf{k}_{2}, \omega_{2}\right)\right\rangle_{0}^{\mathrm{c}}
\end{aligned}
$$

where as usual only connected diagrams are included, the transformation $\mathbf{k}_{2} \rightarrow \mathbf{k}_{2}-\mathbf{k}_{1}, \omega_{2} \rightarrow \omega_{2}-\omega_{1}$ was performed in the final step and $\epsilon(\mathbf{k})=\mu_{V}+\frac{\hbar^{2} \mathbf{k}^{2}}{2 m^{*}}$ is the vison energy. In the Coulomb gauge $\mathbf{k}_{2} \cdot \mathbf{A}\left(\mathbf{k}_{2}, \omega_{2}\right)=0$, and we can write $\mathbf{A}\left(\mathbf{k}_{2}, \omega_{2}\right)=\sqrt{a_{0}} \sum_{\lambda=1,2} A_{\lambda}\left(\mathbf{k}_{2}, \omega_{2}\right) \hat{\mathbf{n}}_{\lambda}\left(\mathbf{k}_{2}\right)$, where $\hat{\mathbf{n}}_{\lambda}(\mathbf{k}) \cdot \hat{\mathbf{n}}_{\lambda^{\prime}}(\mathbf{k})=\delta_{\lambda \lambda^{\prime}}$ and $\hat{\mathbf{n}}_{\lambda}(\mathbf{k}) \cdot \mathbf{k}=0$. This allows us to rewrite the correction to the gauge field correlator as

$$
\begin{aligned}
\delta\left\langle A_{\lambda}(\mathbf{k}, \omega) A_{\lambda^{\prime}}\left(\mathbf{k}^{\prime}, \omega^{\prime}\right)\right\rangle \\
=\frac{16 \pi v^{2}}{\beta} \sum_{\omega_{1}, \omega_{2}} \sum_{\lambda_{1}, \lambda_{2}} \int \frac{d^{3} \mathbf{k}_{1} d^{3} \mathbf{k}_{2}}{(2 \pi)^{6}} \\
\quad \times \frac{\left(a_{0}^{3} / 8\right)\left(\mathbf{k}_{1} \cdot \hat{\mathbf{n}}_{\lambda_{1}}\left(\mathbf{k}_{2}\right)\right)}{-i \omega_{1}+\epsilon\left(\mathbf{k}_{1}\right)} \frac{\left(a_{0}^{3} / 8\right)\left(\mathbf{k}_{1} \cdot \hat{\mathbf{n}}_{\lambda_{2}}\left(\mathbf{k}_{2}\right)\right)}{i \omega_{1}-i \omega_{2}+\epsilon\left(\mathbf{k}_{2}-\mathbf{k}_{1}\right)} \\
= \\
\times a_{0}\left\langle A_{\lambda}(\mathbf{k}, \omega) A_{\lambda^{\prime}}\left(\mathbf{k}^{\prime}, \omega^{\prime}\right) A_{\lambda_{1}}\left(\mathbf{k}_{2}, \omega_{2}\right) A_{\lambda_{2}}\left(-\mathbf{k}_{2},-\omega_{2}\right)\right\rangle_{0}^{\mathrm{c}} \\
=(2 \pi)^{3} \delta^{3}\left(\mathbf{k}+\mathbf{k}^{\prime}\right) \delta_{\omega,-\omega^{\prime}} \delta_{\lambda \lambda^{\prime}} \\
\times \frac{\pi v^{2} a_{0}^{7} \tilde{g}^{2}}{\left(\omega^{2}+c^{2} \hbar^{2} \mathbf{k}^{2}\right)^{2}} \int \frac{d^{3} \mathbf{k}_{1}}{(2 \pi)^{3}} \frac{\mathbf{k}_{1}^{2}-\left(\mathbf{k}_{1} \cdot \hat{\mathbf{k}}\right)^{2}}{\epsilon\left(\mathbf{k}_{1}\right)+\epsilon\left(\mathbf{k}-\mathbf{k}_{1}\right)-i \omega},
\end{aligned}
$$

where by symmetry the integral over $\mathbf{k}_{1}$ vanishes when $\lambda_{1} \neq$ $\lambda_{2}$. The correction to the imaginary-time magnetic field correlator, defined in Eq. (67), is given by

$$
\begin{aligned}
\delta \chi_{\lambda}(\mathbf{k}, i \omega)= & \int \frac{d^{3} \mathbf{k}_{1}}{(2 \pi)^{3}} \frac{\mathbf{k}_{1}^{2}-\left(\mathbf{k}_{1} \cdot \hat{\mathbf{k}}\right)^{2}}{\epsilon\left(\mathbf{k}_{1}\right)+\epsilon\left(\mathbf{k}-\mathbf{k}_{1}\right)-i \omega} \\
& \times \frac{\pi v^{2} a_{0}^{7} \tilde{g}^{2} \xi_{\lambda}^{2}(\mathbf{k})}{\left(\omega^{2}+c^{2} \hbar^{2} \mathbf{k}^{2}\right)^{2}}
\end{aligned}
$$

and the spectral density by

$$
\begin{aligned}
\delta \rho_{\lambda}(\mathbf{k}, \omega)= & \operatorname{Im} \lim _{\epsilon \rightarrow 0} \delta \chi_{\lambda}(\mathbf{k}, \omega+i \epsilon) \\
= & \int \frac{d^{3} \mathbf{k}_{1}}{(2 \pi)^{3}}\left(\mathbf{k}_{1}^{2}-\left(\mathbf{k}_{1} \cdot \hat{\mathbf{k}}\right)^{2}\right) \\
& \times \delta\left(\epsilon\left(\mathbf{k}_{1}\right)+\epsilon\left(\mathbf{k}-\mathbf{k}_{1}\right)-\omega\right) \frac{\pi v^{2} a_{0}^{7} \tilde{g}^{2} \xi_{\lambda}^{2}(\mathbf{k})}{\left(\omega^{2}+c^{2} \hbar^{2} \mathbf{k}^{2}\right)^{2}} \\
= & \frac{\left(m^{*}\right)^{\frac{5}{2}} v^{2} a_{0}^{7} \tilde{g}^{2} \xi_{\lambda}^{2}(\mathbf{k}) \Theta(W) W^{\frac{3}{2}}}{6 \pi \hbar^{5}\left(\omega^{2}+c^{2} \hbar^{2} \mathbf{k}^{2}\right)^{2}},
\end{aligned}
$$

where $W=\omega-2 \mu_{V}-\frac{\hbar^{2} \mathbf{k}^{2}}{4 m^{*}}$ and $\Theta(W)$ is the Heaviside step function. The contribution to the total unpolarized scattering intensity $I(\mathbf{k}, \omega)$ from the generation of vison pairs can now be found using Eq. (72) and the fact that at zero temperature the dynamical structure factor $S_{\lambda}(\mathbf{k}, \omega)=2 \rho_{\lambda}(\mathbf{k}, \omega)$.

\section{APPENDIX E: HEAT CAPACITY}

Integrating out gauge fields $A_{\lambda=1,2}(\mathbf{k}, \omega)$ from the action in Eq. (64) gives us the photon contribution to the partition function

$$
Z_{\mathrm{P}}=\prod_{\omega, \mathbf{k}, \lambda=1,2} \sqrt{\frac{\pi}{\tilde{g} \beta}} \frac{\sqrt{\pi / \beta}}{\sqrt{\frac{\tilde{g} z}{s^{2}}\left|\xi_{\lambda}(\mathbf{k})\right|^{2}+\frac{\omega_{p}^{2}}{4 \tilde{g}}\left(1-\delta_{\omega, 0}\right)+\frac{\omega^{2}}{4 \tilde{g}}}}
$$

where the factor of $\sqrt{\frac{\pi}{\tilde{g} \beta}}$ is a result of previously integrating out the $E_{\lambda=1,2}(\mathbf{k}, \omega)$ fields from the action in Eq. (13). Ignoring temperature-independent prefactors in the partition function and performing the sum over Matsubara frequencies we obtain the photon contribution to the free energy

$$
F_{\mathrm{P}}=k_{B} \sum_{\mathbf{k}, \lambda=1,2}\left(\frac{E_{\mathbf{k}}}{2}+T \ln \left(1-e^{-E_{\mathbf{k}} / T}\right)-T \ln E_{\mathbf{k}}\right) .
$$

The photon contribution to the heat capacity per unit volume is then given by

$$
\begin{aligned}
C_{\mathrm{P}}= & -\frac{T}{V} \frac{\partial^{2} F_{\mathrm{P}}}{\partial T^{2}} \\
= & \frac{k_{B}}{V} \sum_{\mathbf{k}, \lambda=1,2}\left[\frac{\left(T E_{\mathbf{k}}^{\prime}-E_{\mathbf{k}}\right)^{2} e^{E_{\mathbf{k}} / T}}{T^{2}\left(e^{E_{\mathbf{k}} / T}-1\right)^{2}}-\frac{T E_{\mathbf{k}}^{\prime \prime}}{2}\right. \\
& \left.-\frac{T E_{\mathbf{k}}^{\prime \prime}}{e^{E_{\mathbf{k}} / T}-1}+\frac{2 T E_{\mathbf{k}}^{\prime}}{E_{\mathbf{k}}}+\frac{T^{2} E_{\mathbf{k}}^{\prime \prime}}{E_{\mathbf{k}}}-\frac{T^{2}\left(E_{\mathbf{k}}^{\prime}\right)^{2}}{E_{\mathbf{k}}^{2}}\right],
\end{aligned}
$$

where the primes indicate temperature derivatives and the volume $V=\frac{N_{s} a_{0}^{3}}{4}$.

At low temperatures the vison contribution to the partition function is given by

$$
Z_{\mathrm{V}}=\sum_{n} \frac{e^{-n \beta \mu_{V}}}{n !}\left(\frac{V}{\lambda_{B}^{3}}\right)^{n}
$$

The contribution to the heat capacity per unit volume is given by

$$
\begin{aligned}
C_{\mathrm{V}} & =\frac{T^{2}}{V} \frac{\partial^{2} \ln Z_{\mathrm{V}}}{\partial T^{2}} \\
& =\frac{1}{4} \lambda_{B}^{2} \sqrt{T} e^{-\beta \mu_{V}}\left(4 \mu^{2}+12 \mu T+15 T^{2}\right) .
\end{aligned}
$$

\section{APPENDIX F: CONFINEMENT OF SPINONS}

In this section, we demonstrate how a nondynamical mass gap, i.e., one that couples to the zero Matsubara frequency component, would generate confinement. 
We introduce two oppositely charged monopoles situated on up tetrahedra with centres $\mathbf{r} \pm N\left(\mathbf{e}_{1}-\mathbf{e}_{2}\right)$. This results in a line of background flux linking them

$$
\begin{aligned}
\sqrt{N_{s}} B_{\lambda}^{0}(\mathbf{k})= & \sum_{\mathbf{r}, \mu} e^{-i \mathbf{k} \cdot\left(\mathbf{r}+\mathbf{e}_{\mu} / 2\right)} U_{\lambda \mu}(\mathbf{k}) B_{\mu}\left(\mathbf{r}+\mathbf{e}_{\mu} / 2\right) \\
= & \sum_{n=-N}^{N}\left(U_{\lambda 1}(\mathbf{k}) e^{-i \mathbf{k} \cdot\left(n \mathbf{a}+\mathbf{e}_{1} / 2\right)}-U_{\lambda 2}(\mathbf{k})\right. \\
& \left.\times e^{-i \mathbf{k} \cdot\left(n \mathbf{a}+\mathbf{e}_{2} / 2\right)}\right) \\
= & \left(U_{\lambda 1}(\mathbf{k}) e^{-i \mathbf{k} \cdot \mathbf{e}_{1} / 2}-U_{\lambda 2}(\mathbf{k}) e^{-i \mathbf{k} \cdot \mathbf{e}_{2} / 2}\right) \\
& \times \frac{\sin \mathbf{k} \cdot \mathbf{a}\left(N+\frac{1}{2}\right)}{\sin \mathbf{k} \cdot \mathbf{a}}
\end{aligned}
$$

where $\mathbf{a}=\mathbf{e}_{1}-\mathbf{e}_{2}$. By Eq. (8), the noninteger background vector potential is then given by $A_{\lambda}^{0}(\mathbf{k})=B_{\lambda}^{0}(\mathbf{k}) / \xi_{\lambda}(\mathbf{k})$ for $\lambda=1$ and 2. In its presence, the static part of the action in Eq. (64) becomes

$$
S / \beta=\sum_{\mathbf{k}, \lambda=1,2} \frac{\tilde{g} z a_{0}^{2}}{s^{2}}\left(\mathbf{k}^{2}\left|A_{\lambda}(\mathbf{k})+A_{\lambda}^{0}(\mathbf{k})\right|^{2}+\xi^{-2}\left|A_{\lambda}(\mathbf{k})\right|^{2}\right),
$$

where $\xi^{-2}$ is a static mass gap that could be generated by vison condensation in the ground state. Integrating out the field $A_{\lambda}(\mathbf{k})$, we obtain

$$
S / \beta=\frac{\tilde{g} z a_{0}^{2}}{s^{2}} \sum_{\mathbf{k}, \lambda} \frac{\mathbf{k}^{2} \xi^{-2}}{\mathbf{k}^{2}+\xi^{-2}}\left|A_{\lambda}^{0}(\mathbf{k})\right|^{2} .
$$

We can see that in the absence of a static mass gap, i.e., in the limit $\xi \rightarrow \infty$, there is no energy cost arising from the transverse part of the background field $B_{\lambda}^{0}(\mathbf{k})$. There is only a Coulombic energy cost arising from its longitudinal part, i.e., $\Delta_{i j} \psi$ in Eq. (8). Substituting in for $A_{\lambda}^{0}(\mathbf{k})$ and integrating along $\mathbf{k} \cdot \hat{\mathbf{a}}$ first, we obtain

$$
\begin{aligned}
S / \beta= & \frac{\tilde{g} z a_{0}^{3} \pi n}{|\mathbf{a}|} \int_{|\mathbf{k}|<\Lambda} \frac{d^{3} \mathbf{k}}{(2 \pi)^{3}} \frac{\xi^{-2}}{\mathbf{k}^{2}+\xi^{-2}} \frac{|\mathbf{a}| \sin ^{2} n \mathbf{k} \cdot \mathbf{a}}{\pi n(\mathbf{k} \cdot \mathbf{a})^{2}} \\
& \times\left|U_{\lambda 1}(\mathbf{k}) e^{-i \mathbf{k} \cdot \mathbf{e}_{1} / 2}-U_{\lambda 2}(\mathbf{k}) e^{-i \mathbf{k} \cdot \mathbf{e}_{2} / 2}\right|^{2} \\
= & \frac{2 \pi \tilde{g} z a_{0}^{3} n}{|\mathbf{a}|} \int_{|\mathbf{k}|<\Lambda} \frac{d^{2} \mathbf{k}}{(2 \pi)^{3}} \frac{\xi^{-2}}{\mathbf{k}^{2}+\xi^{-2}} \\
\approx & n \frac{\tilde{g} z\left(a_{0} / \xi\right)^{2} \ln \Lambda \xi}{\sqrt{2} \pi}
\end{aligned}
$$

where we have worked in the limit $\Lambda a_{0} \ll 1$ and used the fact that $\lim _{n \rightarrow \infty}\left(\frac{|\mathbf{a}|}{\pi n} \frac{\sin ^{2} n \mathbf{k} \cdot \mathbf{a}}{(\mathbf{k} \cdot \mathbf{a})^{2}}\right)=\delta(\mathbf{k} \cdot \hat{\mathbf{a}})$ and $\mid U_{\lambda 1}(\mathbf{k}) e^{-i \mathbf{k} \cdot \mathbf{e}_{1} / 2}-$
$\left.U_{\lambda 2}(\mathbf{k}) e^{-i \mathbf{k} \cdot \mathbf{e}_{2} / 2}\right|^{2}=2$ at $\mathbf{k} \cdot \hat{\mathbf{a}}=0$. The above result shows that a static mass gap generates a constant confining force equal to

$$
F=\frac{\tilde{g} z\left(a_{0} / \xi\right)^{2} \ln \Lambda \xi}{\sqrt{2} \pi a_{0}} .
$$

\section{APPENDIX G: PLASMA OSCILLATIONS IN THE CONTINUUM LIMIT}

We begin with the action in Eq. (13) and take the continuum limit as follows:

$$
\begin{aligned}
& A_{\alpha \beta}(\tau) \rightarrow \mathbf{A}(\mathbf{r}, \tau) \cdot\left(\mathbf{r}_{\beta}-\mathbf{r}_{\alpha}\right), \\
& E_{\alpha \beta}(\tau) \rightarrow \mathbf{E}(\mathbf{r}, \tau) \cdot\left(\mathbf{r}_{\beta}-\mathbf{r}_{\alpha}\right), \\
& \mathcal{S}=\int_{0}^{\beta} d \tau \int \frac{d^{3} \mathbf{r}}{a_{0}}\left(i \mathbf{E} \cdot \dot{\mathbf{A}}+\tilde{g} \mathbf{E}^{2}+\frac{\tilde{g} z a_{0}^{2}}{s^{2}}(\nabla \times \mathbf{A})^{2}\right) .
\end{aligned}
$$

We can also consider adding a vison current term to the action which couples to the magnetic vector potential

$$
\delta \mathcal{S}=-\int d^{3} \mathbf{r} \mathbf{J} \cdot \mathbf{A} .
$$

Moving from imaginary to real time (which we measure in units of $\hbar$ ), we obtain the saddle point equations of the action, i.e., Maxwell's equations

$$
\begin{aligned}
\dot{\mathbf{A}} & =-2 \tilde{g} \mathbf{E}, \\
\dot{\mathbf{E}} & =\frac{2 \tilde{g} a_{0}^{2} z}{s^{2}} \nabla \times(\nabla \times \mathbf{A})-\mathbf{J} a_{0} .
\end{aligned}
$$

In the Debye limit $e^{\beta \mu_{V}} \gg 1$, when there are many visons inside the Debye volume, the visons will respond to the gauge field coherently and we can use a hydrodynamic description. The charges accelerate in the presence of the electric field according to

$$
\dot{\mathbf{J}}=-2(2 \pi \hbar)^{2} n \dot{\mathbf{A}} / m^{*},
$$

where $n=e^{-\beta \mu_{V}} \lambda_{B}^{-3}$ is the vison number density and $(2 \pi \hbar)$ is the vison charge. Differentiating the second equation in Eq. (G3) with respect to time, substituting in for $\dot{\mathbf{A}}$ with the help of the first equation and using $\dot{\mathbf{J}}=-2(2 \pi \hbar)^{2} n \dot{\mathbf{A}} / m^{*}$, we obtain the plasma equation

$$
\hbar^{2} c^{2} \nabla^{2} \mathbf{E}=\ddot{\mathbf{E}}+\omega_{p}^{2} \mathbf{E},
$$

where $c^{2}=\frac{4 \tilde{g}^{2} a_{0}^{2} z}{\hbar^{2} s^{2}}, \omega_{p}^{2}=\frac{16 \pi^{2} \hbar^{2} a_{0} e^{-\beta \mu_{V}} \tilde{g}}{m^{*} \lambda_{B}^{3}}$, and we have assumed plasma neutrality so that $\nabla \cdot \mathbf{E}=-\nabla^{2} \varphi=0$ everywhere. This result agrees with the derivation presented in the main body of this paper.
[1] M. J. P. Gingras and P. A. McClarty, Rep. Prog. Phys. 77, 056501 (2014).

[2] J. Knolle and R Moessner, Annu. Rev. Condens. Matter Phys. 10, 451 (2019).

[3] A. Kitaev, Ann. Phys. 321, 2 (2006).
[4] A. Banerjee, C. A. Bridges, J.-Q. Yan, A. A. Aczel, L. Li, M. B. Stone, G. E. Granroth, M. D. Lumsden, Y. Yiu, J. Knolle, S. Bhattacharjee, D. Kovrizhin, R. Moessner, D. A. Tennant, D. G. Mandrus, and S. E. Nagler, Nat. Mater. 15, 733 (2016). 
[5] A. Banerjee, J. Yan, J. Knolle, C. A. Bridges, M. B. Stone, M. D. Lumsden, D. G. Mandrus, D. A. Tennant, R. Moessner, and S. E. Nagler, Science 356, 1055 (2017).

[6] S.-H. Do, S.-Y. Park, J. Yoshitake, J. Nasu, Y. Motome, Y. S. Kwon, D. T. Adroja, D. J. Voneshen, K. Kim, T.-H. Jang, J.-H. Park, K.-Y. Choi, and S. Ji, Nat. Phys. 13, 1079 (2017).

[7] A. Banerjee, P. Lampen-Kelley, J. Knolle, C. Balz, A. A. Aczel, B. Winn, Y. Liu, D. Pajerowski, J. Yan, C. A. Bridges, A. T. Savici, B. C. Chakoumakos, M. D. Lumsden, D. A. Tennant, R. Moessner, D. G. Mandrus, and S. E. Nagler, Quantum Mater. 3, 8 (2018).

[8] Y. Kasahara, T. Ohnishi, Y. Mizukami, O. Tanaka, S. Ma, K. Sugii, N. Kurita, H. Tanaka, J. Nasu, Y. Motome, T. Shibauchi, and Y. Matsuda, Nature (London) 559, 227 (2018).

[9] M. P. Kwasigroch, B. Douçot, and C. Castelnovo, Phys. Rev. B 95, 134439 (2017).

[10] M. Hermele, M. P. A. Fisher, and L. Balents, Phys. Rev. B 69, 064404 (2004).

[11] A. M. Polyakov, Nucl. Phys. B 120, 429 (1977)

[12] G. Chen, Phys. Rev. B 94, 205107 (2016).

[13] G. Chen, Phys. Rev. B 96, 195127 (2017).

[14] O. Benton, O. Sikora, and N. Shannon, Phys. Rev. B 86, 075154 (2012).

[15] P. C. Kwok and T. D. Schultz, J. Phys. C: Solid State Phys. 2, 1196 (1969).

[16] N. Shannon, O. Sikora, F. Pollmann, K. Penc, and P. Fulde, Phys. Rev. Lett. 108, 067204 (2012).

[17] Y. Kato and S. Onoda, Phys. Rev. Lett. 115, 077202 (2015).

[18] A. Banerjee, S. V. Isakov, K. Damle, and Y. B. Kim, Phys. Rev. Lett. 100, 047208 (2008).

[19] J.-P. Lv, G. Chen, Y. Deng, and Z. Y. Meng, Phys. Rev. Lett. 115, 037202 (2015).
[20] C.-J. Huang, Y. Deng, Y. Wan, and Z. Y. Meng, Phys. Rev. Lett. 120, 167202 (2018).

[21] L. Savary and L. Balents, Phys. Rev. Lett. 108, 037202 (2012).

[22] S. B. Lee, S. Onoda, and L. Balents, Phys. Rev. B 86, 104412 (2012).

[23] L. Savary and L. Balents, Phys. Rev. B 87, 205130 (2013).

[24] Z. Hao, A. G. R. Day, and M. J. P. Gingras, Phys. Rev. B 90, 214430 (2014).

[25] O. Benton, L. D. C. Jaubert, R. R. P. Singh, J. Oitmaa, and N. Shannon, Phys. Rev. Lett. 121, 067201 (2018).

[26] A. Szabó and C. Castelnovo, Phys. Rev. B 100, 014417 (2019).

[27] A. M. Polyakov, Phys. Lett. B 72, 477 (1978).

[28] J. Villain, J. Phys. 35, 27 (1974).

[29] L. D. Faddeev and V. N. Popov, Phys. Lett. B 25, 29 (1967).

[30] J. M. Kosterlitz, J. Phys. C 10, 3753 (1977).

[31] In this limit, the typical instanton separation along $\tau$ will be small by comparison with $\beta$, so that finite-size effects associated with finite temperature are negligible.

[32] Consider a pair of tightly bound visons of opposite charge, where one of them executes two instanton hops returning to its original position. Because, there is a growing energy cost associated with separating the two instanton hops in imaginary time, the oscillation will not generate a mass gap but only renormalize the speed of light.

[33] T. Fennell, P. P. Deen, A. R. Wildes, K. Schmalzl, D. Prabhakaran, A. T. Boothroyd, R. J. Aldus, D. F. McMorrow, and S. T. Bramwell, Science 326, 415 (2009).

[34] D. Khomskii, Nat. Commun. 3, 904 (2012).

[35] E. Lantagne-Hurtubise, S. Bhattacharjee, and R. Moessner, Phys. Rev. B 96, 125145 (2017).

[36] S. Nakosai and S. Onoda, J. Phys. Soc. Jpn. 88, 053701 (2019). 